\title{
THE GEOMETRY AND THE ANALYTIC PROPERTIES OF ISOTROPIC MULTIRESOLUTION ANALYSIS
}

\author{
JUAN R. ROMERO, SIMON K. ALEXANDER, SHIKHA BAID, SAURABH JAIN, AND MANOS PAPADAKIS
}

\begin{abstract}
In this paper we investigate Isotropic Multiresolution Analysis (IMRA), isotropic refinable functions, and wavelets. The main results are the characterization of IMRAs in terms of the Lax-Wiener Theorem, and the characterization of isotropic refinable functions in terms of the support of their Fourier transform. As an immediate consequence of these results, there are no compactly supported (in the space domain) isotropic refinable functions in many dimensions. Next we study the approximation properties of IMRAs. Finally, we discuss the application of IMRA wavelets to 2D and 3D-texture segmentation in natural and biomedical images.
\end{abstract}

\section{INTRODUCTION}

The main goals of this paper are to introduce a new type of multiresolution analysis (MRA) which has a particular type of isotropy (namely its 'core' subspace $V_{0}$ is invariant under rotations) and to prove that there are no radial or even further isotropic refinable functions with compact support in $L^{2}\left(\mathbb{R}^{d}\right)$. Furthermore, we study the properties of these Isotropic MRAs and refinable functions, and develop certain classes of them. The driving application of this work is 3D-texture segmentation with emphasis in biomedical imaging. We note that we use the term MRA in this paragraph with some freedom hoping that we are not ambiguous.

1.1. Background. Our motivation to introduce and study IMRAs and isotropic refinable functions came from the need to use filters with a high degree of symmetry ideally isotropic in order to eliminate directional bias in digital filtering. Here and hereafter we use the term 'filtering directional bias' without attempting to define it. Neither there exists a formal definition of this term in the literature nor we are the first who realized the need to reduce this sort of filtering bias. In fact, this need has motivated the constructions of filters and multiresolution analyses that are not tensor products of one-dimensional ones. The main drawback of the tensor products of the 1D-MRAs is that the resulting image processing is carried out in a row and column fashion [67, p. 410]. The acknowledgment of this drawback led to the development of non-separable MRAs e.g. [4-8, 10, 18, 23, 28, 39, 42, 49, 51]. In addition, almost all of these constructs are in two-dimensions. However, the scaling functions of the non-separable MRA designs suffer from lack of either symmetry or smoothness or both (see the introduction of [10]). The main impediment in generating scaling functions in higher dimensions that are not tensor products of one-dimensional scaling functions is that the Fejér-Riesz spectral factorization theorem [44] cannot be generalized in more than one dimensions.

An alternative method to reduce the effects of directional bias is to introduce more preferred directions for the filtering by augmenting one dimensional constructions with multiscale or monoscale angular decomposition. In other words one might spread this sort of bias into more than the obvious directions. This idea leads to the so-called directional representations. There are two schools that follow this approach: One pioneered by Candes, Donoho and collaborators who introduced curvelets, ridgelets, wedgelets, beamlets, planelets, sheerlets, etc. (e.g. see $[19,29,30,40,50,60])$. Alternative constructs with monoscale angular resolution have also been proposed $[26,48,62,63]$. Other directional representations in the spirit of digital filter design, not directly related to multiresolution analyses nor to wavelets can be found in $[1,22,36,43,45,47,59,64,66,68]$.

Key words and phrases. Isotropic wavelets, Isotropic multiresolution, 3D-textures, steerability, 3D-data representations, Rigid motion covariant representations.

J.R. Romero is with the University of Puerto Rico at Mayagüez, Department of Mathematical Sciences. S.K. Alexander, S. Baid, S. Jain and M. Papadakis are with the University of Houston, Department of Mathematics. 
Precisely for the reasons stated above, isotropic filters have been used in the past - not in an MRAcontext but for multiscale transforms. The most well known of them and probably the oldest is the Canny Edge Detector [20] motivated by the studies of Marr and Hilldreth [54] on vision. More recently, the use of isotropic filters or scaling functions has been proposed by several authors. Starck uses them to analyze e.g. astronomical images of objects with a high degree of spherical symmetry e.g., [65], while others use them to construct covariant representations with respect to rigid motions: Among them Fickus and co-workers develop higher-order moments covariant with respect to rigid motions in multidimensions [33,34]. Our approach to isotropic filters and IMRAs originated in [58] and continued in [15,57] and the present paper.

The need to derive MRA wavelet decompositions covariant with respect to rigid motions also motivated $[24,35]$. However, these constructs are in 2D and are not generalizable to higher dimensions and the Fourier transforms of their radial Riesz scaling functions are discontinuous at the origin.

Other notable constructions of multidimensional, possibly non-MRA smooth, isotropic, frame wavelets have been proposed in $[2,31,32,41,55]$. However, these have not been shown to arise from MRAs or yield decompositions implementable with MRA-type fast wavelet algorithms.

The driving application for our study of IMRAs is tissue identification in biomedical imaging [15, 37, 38, 57]. Specifically in our initial work the input 3D-data sets are $\mu C T$ (micro Computed Tomography) images of arterial specimens. To each voxel we assign one out of a total of three labels corresponding to three types of tissue one of which is normal and two are abnormal, but the problem is more general and highly complex.

Generally speaking, a typical biomedical image of the type we are interested in will be a set of scalar valued measurements on a square lattice in two or three dimensions (referred to as pixels and voxels, respectively). The physical relevance of the values depends on the modality, or type of acquisition device. Typically, values will be constrained to integers representable in some number of bits, i.e. integer values in the interval $\left[0,2^{n}-1\right]$ or perhaps $\left[-2^{m}-1,2^{m}-1\right]$, for integers $n, m$ which will typically be values $\leq 16$. Examples of such images are given in Figure 1.

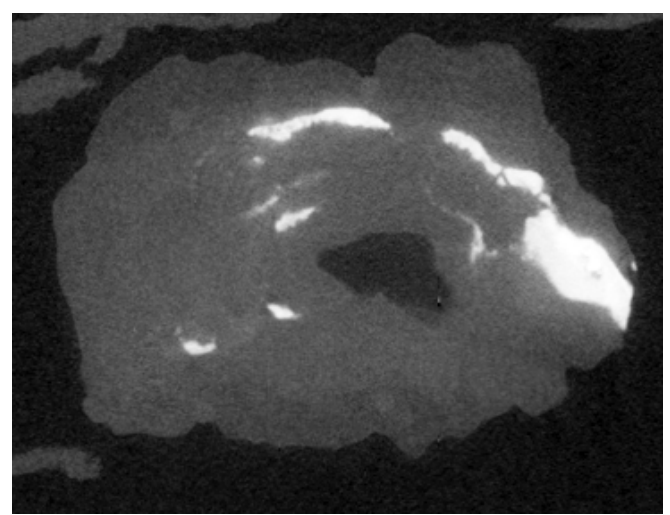

(a) $2 \mathrm{D}$ slice from $3 \mathrm{D} \mu \mathrm{CT} \mathrm{x}$-ray data

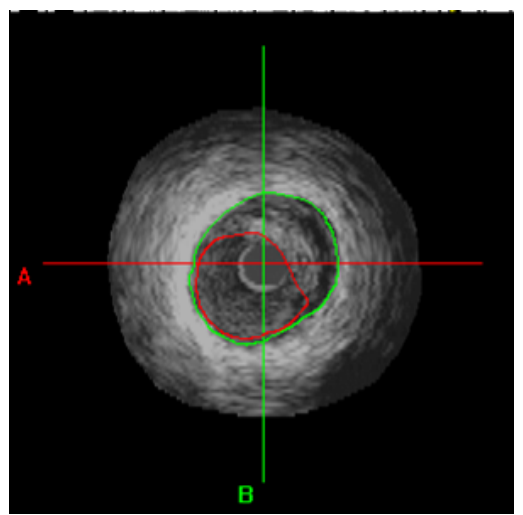

(b) slice from Intravascular Ultra Sound data

FIGURE 1. Examples of medical 3D data sets.

Our approach to tissue segmentation/identification in 3D-medical data sets is based on the belief that different tissue types correspond to different 3D-textures, due to plain tissue differentiation. Thus we view the problem of tissue segmentation/identification as a problem of 3D-texture segmentation/identification. Texture segmentation/identification requires two major components, developed together; the representation of the original data and the segmentation/identification algorithm. We model textures as random variables to address variability due to intra-tissue type natural variation and due to noise. However, in this work we neither touch the probabilistic part nor the algorithmics of 3D-texture segmentation/identification. We limit our discussion on texture representations based on IMRAs and we introduce a generic model for 3D-data representations that can lead to a 3D-rigid motion invariant texture segmentation/identification (Section 7). 
The IMRA-based representation may not be the most appropriate for all textures in the following intuitive sense: It appears to be most suitable for textures which do not exhibit only a small number of preferred directions (e.g. the arterial 'normal' tissue in Fig. 1-a, ignoring the bright calcium spots); for those that do exhibit preferred directions texture encoding by means of directional representations may well be sparser. We note that the former case (no preferred direction) is quite typical in many medical tissue segmentation problems, so restricting our focus here is not particularly narrow. As is typical for segmentation, 'tuning' the algorithmic approach to the specifics of the particular application gives improved results.

The rest of the paper is organized as follows: In Sections 2 and 3 we characterize IMRAs and isotropic refinable functions respectively. In the latter section we construct isotropic wavelet Parseval frames arising from them. To facilitate fast algorithmic implementations of IMRA decompositions and reconstructions [15] we revisit the so-called Extension Principles (Section 4). We construct classes of IMRAs, their isotropic refinable functions, filters and wavelets in Section 5. The approximation properties of IMRAs are studied in Section 6. In Section 7 we propose a generic mathematical model for 3D-data representations that can lead to a 3D-rigid motion invariant texture segmentation/identification and we discuss how IMRA-based data representations fit into this model and why can be useful for tissue segmentation/identification in certain classes of medical imaging data.

1.2. Notation and Preliminaries. The Fourier transform $\widehat{f}: \mathbb{R}^{d} \longrightarrow \mathbb{C}$ of $f \in L^{1}\left(\mathbb{R}^{d}\right)$ is defined by

$$
\forall \xi \in \mathbb{R}^{d}, \widehat{f}(\xi)=\int_{\mathbb{R}^{d}} f(x) e^{-2 \pi i x \cdot \xi} d x .
$$

Here, $x \cdot \xi$ denotes the standard inner product on $\mathbb{R}^{d} \times \mathbb{R}^{d}$. The map $f \rightarrow \widehat{f}$ restricted to $L^{1}\left(\mathbb{R}^{d}\right) \cap L^{2}\left(\mathbb{R}^{d}\right)$ extends to a unitary map on $L^{2}\left(\mathbb{R}^{d}\right)$.

A frame for a separable Hilbert space $H$ is a sequence $\left\{f_{i}\right\}_{i \in \mathbb{I}} \subseteq H$, where $\mathbb{I}$ is a countable index set, for which there are constants $A, B>0$ such that

$$
\forall f \in H, A\|f\|^{2} \leq \sum_{i \in \mathbb{I}}\left|\left\langle f, f_{i}\right\rangle\right|^{2} \leq B\|f\|^{2} .
$$

$A$ and $B$ are called frame constants. The weaker version of the above inequality where $B>0$ such that the right-hand side of the above inequality is valid for all $f \in \mathbb{R}^{d}$ is termed by referring to $\left\{f_{i}\right\}_{i \in \mathbb{I}}$ as a Bessel sequence. If $A=B$, we say the frame is tight, and a tight frame with $A=B=1$ is called Parseval. A frame is a Riesz basis for $H$ if it is also a minimal family generating $H$. We use the terms frame (Riesz) sequence when we refer to a countable frame or a Riesz basis of a subspace of a Hilbert space.

For $y \in \mathbb{R}^{d}$, the (unitary) shift operator $T_{y}$ is defined as $T_{y} f(x)=f(x-y), f \in L^{2}\left(\mathbb{R}^{d}\right)$. Given a lattice $\Gamma \subset \mathbb{R}^{d}$ which is similar to $\mathbb{Z}^{d}$, a linear closed subspace $V$ of $L^{2}\left(\mathbb{R}^{d}\right)$ is said to be $\Gamma$-shift-invariant if for each $\gamma \in \Gamma, T_{\gamma} V=V$. It is well-known that every $\Gamma$-shift-invariant subspace $V$ contains a denumerable set Parseval frame of itself. When we use the term shift-invariant for a subspace we mean $\Gamma=\mathbb{Z}^{d}$. Given $S$, a subset of a linear space $V$, the notation $\langle S\rangle$ will be used for the shift invariant subspace generated by $S$ (i.e., the smallest shift invariant subspace of $V$ containing S). If $S=\{\phi\}$, we will write $\langle\phi\rangle$ to denote $\langle\{\phi\}\rangle$.

Moreover, $\mathbb{S}^{d-1}$ denotes the $d$-dimensional unit sphere centered at the origin. For $x \in \mathbb{R}^{d}$ and $\rho>0$, $B(x, \rho)$ will denote the ball centered at $x$ with radius $\rho$. We will not distinguish open from closed balls because the difference $\overline{B(x, \rho)} \backslash B(x, \rho)$ (a $d$ sphere) has measure zero in $\mathbb{R}^{d}$. By $\operatorname{supp} f$ we mean the set of points $x$ such that $f(x) \neq 0$.

Given $\Omega \subset \mathbb{R}^{d}$ with positive measure, $P W_{\Omega}$ is the closed subspace of $L^{2}\left(\mathbb{R}^{d}\right)$ defined as

$$
P W_{\Omega}=\left\{f \in L^{2}\left(\mathbb{R}^{d}\right): \operatorname{supp} \widehat{f} \subseteq \Omega\right\} .
$$

If $r$ is a positive real number, $P W_{r}$ will denote $P W_{B(0, r)}$. The subspace $P W_{\Omega}$ is called a Paley-Wiener subspace of $L^{2}\left(\mathbb{R}^{d}\right)$ associated to $\Omega$, if $\Omega$ has compact closure, or simply a Wiener subspace of $L^{2}\left(\mathbb{R}^{d}\right)$ associated to $\Omega$ if $\Omega$ is an arbitrary measurable subset of $\mathbb{R}^{d}$. The Lax-Wiener Theorem states that these are the only subspaces of $\mathbb{R}^{d}$ that are invariant under the action the group of translations induced by $\mathbb{R}^{d}$ [52]. 
This theorem plays a central role in the characterization of IMRAs (Section 2). Finally, we remark that all set equalities and inclusions are modulo null sets and $e_{n}(\xi):=e^{-2 \pi i(n \cdot \xi)}$, with $\xi \in \mathbb{R}^{d}$.

Definition 1.1. Let $\Omega$ be a measurable subset of $\mathbb{R}^{d}$.

1. We say that $\Omega \subset \mathbb{R}^{d}$ is radial if for all $O \in S O(d), O \Omega=\Omega$.

2. A $d \times d$ matrix is expansive if it has integer entries and if all of its eigenvalues have absolute value greater than 1.

3. An expansive matrix is radially expansive if $A=a O$, for some fixed $a>0$ and a matrix $O \in$ $S O(d)$.

A radially expansive matrix $A$ is a matrix with integer entries that leaves the lattice $\mathbb{Z}^{d}$ invariant, and $|\operatorname{det} A|$ is a positive integer. The term isotropic is sometimes used to refer to matrices that are similar to a diagonal matrix and for which every eigenvalue has the same absolute value [53]. However, these 'isotropic' matrices are not suitable for our study because they do not necessarily map radial sets into radial sets. We also note that we use the term 'isotropic' in an entirely different context.

If $A$ is a radially expansive matrix, we define its associated unitary dilation operator by

$$
\forall f \in L^{2}\left(\mathbb{R}^{d}\right), D_{A} f(x)=|\operatorname{det} A|^{1 / 2} f(A x)
$$

A function $g$ defined on $\mathbb{R}^{d}$ is said to be radial if, $g\left(x_{1}\right)=g\left(x_{2}\right)$, whenever $\left\|x_{1}\right\|=\left\|x_{2}\right\|$. A subset $\mathfrak{F}$ of $\mathbb{R}^{d}$ is radial if its characteristic function $\chi_{\mathfrak{F}}$ is equal a.e. to a Lebesgue measurable radial function. Now, let $\mathfrak{F}$ be a Lebesgue measurable subset of $\mathbb{R}^{d}$. Then, $\chi_{\mathfrak{F}}$ is measurable and now a change of coordinates from Cartesian to spherical together with Tonelli's theorem readily imply

$$
\int_{\mathbb{R}^{d}} \chi_{\mathfrak{F}}=\int_{0}^{\infty} \int_{\mathbb{S}^{d-1}} \chi_{\mathfrak{F}}(r, \theta) r^{d-1} d \theta d r .
$$

For a.e. $r>0$, the function $\chi_{\mathfrak{F}}(r, \cdot)$ is a.e. constant on the sphere $r \mathbb{S}^{d-1}$, so either it takes the value 1 or the value 0 on $r \mathbb{S}^{d-1}$. But, the result of the first of the two successive integrations is a measurable function with respect to $r$, and the value of this function is either $m\left(\mathbb{S}^{d-1}\right)$ or 0 , where $m\left(\mathbb{S}^{d-1}\right)$ is the area of the sphere $\mathbb{S}^{d-1}$. So, there exists a measurable subset $F$ of $\mathbb{R}^{+}$such that

$$
\int_{\mathbb{R}^{d}} \chi_{\mathfrak{F}}=m\left(\mathbb{S}^{d-1}\right) \int_{0}^{\infty} \chi_{F}(r) r^{d-1} d r .
$$

We will call this set $F$ a radial profile of $\mathfrak{F}$. This set is Lebesgue measurable but it is not uniquely defined for any given radial subset of $\mathbb{R}^{d}$. Nonetheless, all radial profiles are 'equal' in the sense of measure-theoretic set-equality. Generalizing this analysis we call a measurable function $f$ defined on $\mathbb{R}^{d}$ radial, if for almost every $r>0$, the restriction of $f$ on $r \mathbb{S}^{d-1}$ is a.e. equal to a constant function, with respect to the surface measure of $\mathbb{S}^{d-1}$. Thus, from now on, all these functions are considered constant on the spheres $r \mathbb{S}^{d-1}$, for a.e. $r>0$. This also applies to characteristic functions of radial sets. Thus, if $\mathfrak{F}$ is a radial set, then for a.e. $r$ in its radial profile, the sphere $r \mathbb{S}^{d-1}$ is contained in $\mathfrak{F}$. This readily implies that radial sets remain invariant under all rotations and radially expansive matrices map radial sets onto radial sets.

To simplify the language we henceforth drop the term measurable when we refer to functions and sets, since we assume that all of them are measurable. We will often need to consider the restriction of a function on a radial set $\mathfrak{F}$. Doing so, we use the term 'radial' to refer to the property that the restrictions of the function to the spheres $r \mathbb{S}^{d-1}$ are constant for a.e. $r$ in a radial profile of $\mathfrak{F}$.

If $L$ is a measurable subset of $\mathbb{R}^{d}, x_{0}$ is a point of density of $L$ if,

$$
\lim _{\eta \rightarrow 0} \frac{\mid L \cap\left(B\left(x_{0}, \eta\right) \mid\right.}{|B(0, \eta)|}=1 .
$$

A well-known theorem due to Lebesgue asserts that a.e. every point of $L$ is a point of density of $L$, e.g. [69], and for a.e. $x_{0}$ in $L^{c}$

$$
\lim _{\eta \rightarrow 0} \frac{\mid L \cap\left(B\left(x_{0}, \eta\right) \mid\right.}{|B(0, \eta)|}=0 .
$$


Since radial sets remain invariant under rotations we readily assert that if $x_{0}$ is a point of density for some radial set, then all the points on the sphere $\left\|x_{0}\right\| \mathbb{S}^{d-1}$ share the same property. A function $f \in L^{2}\left(\mathbb{R}^{d}\right)$ is said to be isotropic if there exist $y \in \mathbb{R}^{d}$ and a radial function $g \in \mathbb{R}^{d}$ such that $f=T_{y} g$.

We are now ready to introduce the concept of Isotropic Multiresolution Analysis (IMRA). First, this structure must be a multiresolution analysis in the classical sense, without requiring the existence of an orthonormal or Riesz basis for its 'core' subspace $V_{0}$. Therefore, an IMRA is expected to satisfy Properties 1 through 3 of Definition 1.2. Moreover, we require that $V_{0}$ is shift-invariant, in the sense that it remains constant under the action of the group $\left\{T_{n}: n \in \mathbb{Z}^{d}\right\}$ (Property 4 of Definition 1.2), a property that 'core' subspaces of classical MRAs have as well. Therefore, each $V_{j}$ is invariant under the action of the translation group representation of $A^{-j} \mathbb{Z}^{d}$. The problem that concerns wavelet practitioners is the covariance of wavelet representations of images and signals in general under shifts or other transformations of the coordinate system. Specializing this problem to images it is crucial for a significant number of tasks in image analysis to have covariant representations with respect to rigid motions, that is rotations and translations of an image especially in two or three dimensions. Such representations are also known as steerable and there are previous attempts with directional representations to address this problem in the context of digital images for finite subgroups of the rotation group and in two dimensions [1, 22, 36, 43, 45, 47, 59, 64, 66, 68]. Those constructs are not MRA-based and they typically refer to steerability without attempting to define it. To the best of our knowledge, 3D-rigid motion covariant multiscale representations are practically unexplored. Specializing on MRAs, it is natural to impose weaker conditions on the MRA subspaces and in particular on the core subspace $V_{0}$ for MRAs defined with respect to dilations given by radially expansive matrices before even attempting to construct MRA-wavelet decompositions that are covariant with respect to rigid motions. In our first design we used radial ideal filters in order to achieve a high degree of symmetry in wavelet filter design [58], generalizing thus the Shannon MRA (for a definition see [44]) in multidimensions. In order to achieve radiality we relaxed the classical Riesz basis of the integral translates of the scaling function assumption to allow for this set to be a non-minimal frame. All these ideas led us to the addition of the last property in Definition 1.2. This property was originally proposed by Bodmann, Papadakis and Romero for another type of multiresolution analysis customized for spherical coordinates [14]. With these remarks in mind we proceed to the definition an Isotropic Multiresolution Analysis:

Definition 1.2. An Isotropic Multiresolution Analysis (IMRA) of $L^{2}\left(\mathbb{R}^{d}\right)$ with respect to a radially expansive matrix $A$ is a sequence $\left\{V_{j}\right\}_{j \in \mathbb{Z}}$ of closed subspaces of $L^{2}\left(\mathbb{R}^{d}\right)$ satisfying the following conditions:

(1) $\forall j \in \mathbb{Z}, V_{j} \subset V_{j+1}$,

(2) $\left(D_{A}\right)^{j} V_{0}=V_{j}$,

(3) $\cup_{j \in \mathbb{Z}} V_{j}$ is dense in $L^{2}\left(\mathbb{R}^{d}\right)$ and $\cap_{j \in \mathbb{Z}} V_{j}=\{0\}$,

(4) $V_{0}$ is invariant under the action the group $\left\{T_{n}: n \in \mathbb{Z}^{d}\right\}$,

(5) If $P_{0}$ is the orthogonal projection onto $V_{0}$, then

$$
\mathcal{O} P_{0}=P_{0} \mathcal{O} \quad \text { for all } O \in S O(d),
$$

where $\mathcal{O}$ is the unitary operator given by $\mathcal{O} f(x):=f\left(O^{T} x\right)$ a.e. with $f \in L^{2}\left(\mathbb{R}^{d}\right)$ and $O \in S O(d)$ and $O^{T}$ is the transpose of the matrix $O$.

Although the last two properties of Definition 1.2 appear not to be sufficiently strong to yield rigid motion covariant wavelet decompositions, they force all the resolution subspaces $V_{j}$ of an IMRA to be invariant under all rigid motions in any number of dimensions (Theorem 2.1). This strong result follows from the characterization of all IMRAs by means of the Lax-Wiener Theorem [52] which is the topic of the next section. The discussion on the steerability of the IMRA-based wavelet decompositions is postponed for Section 7 where we formally define rigid motion covariant representations. 


\section{CHARACTERIZATION OF IMRAS}

As we will see Equation (1) imposes an algebraic-geometric constraint on the space $V_{0}$ : Assume $V$ is a shift-invariant subspace of $L^{2}\left(\mathbb{R}^{d}\right)$ satisfying (1) as well. Then, for every $O \in S O(d)$ and $n \in \mathbb{Z}^{d}$ we have

$$
T_{n} \mathcal{O}=\mathcal{O} T_{O^{T} n} .
$$

Thus, we obtain $\mathcal{O} T_{O^{T}} V=T_{n} \mathcal{O} V=V$ yielding

$$
T_{O^{T} n} V=V, \text { for every } O \in S O(d) \text { and } n \in \mathbb{Z}^{d} \text {. }
$$

Since $S O(d)$ acts transitively on spheres centered at the origin, the orbit $\{O x: O \in S O(d)\}$ of any $x$ in $\mathbb{R}^{d}$ is the entire sphere of radius $\|x\|$. In particular, if $x=(1,1, \ldots, 1)$, then, Eq. (2) implies that $V$ remains invariant under all translations by $T_{y}$ with $y \in \sqrt{d} \cdot \mathbb{S}^{d-1}$ and thus also by translations $T_{y}$ with $y \in \sqrt{d} \mathbb{S}^{d-1}-\sqrt{d} \mathbb{S}^{d-1}$, which contains the fundamental domain $\mathbb{T}^{d}:=\left[-\frac{1}{2}, \frac{1}{2}\right)^{d}$. Hence, we conclude that $T_{y}(V)=V$ for all $y \in \mathbb{T}^{d}$. Since the same identity is true for all $y \in \mathbb{Z}^{d}$ we assert that $V$ remains invariant under all translations induced by $\mathbb{R}^{d}$. This implies that $V$ is a Wiener subspace of $L^{2}\left(\mathbb{R}^{d}\right)$, so there exists a measurable subset $\Omega$ of $\mathbb{R}^{d}$ such that $V=P W_{\Omega}$. The fact that $V$ remains invariant by all rotations now implies that $\Omega$ is radial. On the other hand, if $\Omega$ is radial we obviously have that $V=P W_{\Omega}$ remains invariant under all rotations and translations induced by $\mathbb{Z}^{d}$. Therefore, we have proved the following characterization of shift-invariant and rotation invariant subspaces of $L^{2}\left(\mathbb{R}^{d}\right)$.

Theorem 2.1. Let $V$ be an invariant subspace of $L^{2}\left(\mathbb{R}^{d}\right)$ under the action of the translation group induced by $\mathbb{Z}^{d}$. Then $V$ remains invariant under all rotations if and only if $V=P W_{\Omega}$ for some radial measurable subset $\Omega$ of $\mathbb{R}^{d}$.

As an immediate consequence we obtain a characterization of IMRAs generated by a single function, in the sense that there exists function $\varphi$ such that $V_{0}=\langle\varphi\rangle$.

Proposition 2.2. Let $A$ be a radially expansive matrix and $C:=A^{*}$. A sequence $\left\{V_{j}\right\}_{j \in \mathbb{Z}}$ is an IMRA with respect to $A$ if and only if $V_{j}=P W_{C^{j} \Omega}$, where $\Omega$ is radial and satisfies

(i) $\Omega \subset C \Omega$.

(ii) The set-theoretic complement of $\cup_{j=1}^{\infty} C^{j} \Omega$ is null.

(iii) $\lim _{j \rightarrow \infty}\left|C^{-j} \Omega\right|=0$.

Moreover the only singly generated IMRAs are precisely $V_{j}=P W_{C^{j} \Omega}$, where $\Omega$ is a radial subset of $\mathbb{T}^{d}$ satisfying (i), (ii) and (iii).

Proof. If $\left\{V_{j}\right\}$ is an IMRA, then $V_{0}$ satisfies equation (1). By the previous theorem, $V_{0}=P W_{\Omega}$ for some set $\Omega \subset \mathbb{R}^{d}$. Thus, $V_{j}=P W_{C^{j} \Omega}$. Properties (i), (ii) and (iii) are now obvious.

On the other hand if $V_{j}=P W_{C^{j} \Omega}$, for some radial subset $\Omega$, and conditions (i), (ii) and (iii) are satisfied, then clearly $\left\{V_{j}\right\}$ is an Isotropic Multiresolution Analysis.

Now, suppose that $V_{0}=\langle\phi\rangle$. Then $\operatorname{supp} \phi=\Omega$ where the equality must be perceived in the measuretheoretic sense. We will prove $\Omega \subseteq \mathbb{T}^{d}$. Since, $\Phi_{\phi}(\xi):=\sum_{k \in \mathbb{Z}^{d}}|\hat{\phi}(\xi+k)|^{2}$ is a.e. finite the function $\omega$ given by $\hat{\omega}:=\hat{\phi} \Phi^{-1 / 2}$ is a Parseval frame generator of $V_{0}$, i.e. $\left\{T_{n} \omega: n \in \mathbb{Z}^{d}\right\}$ is a Parseval frame of $V_{0}$, because $\Phi_{\omega}(\xi):=\sum_{k \in \mathbb{Z}^{d}}|\hat{\omega}(\xi+k)|^{2}=\chi_{\mathcal{E}}$, where $\mathcal{E}$ is a subset of $\mathbb{T}^{d}$ e.g. [12,16,56]. Consequently, there exists a family $\left\{\mathcal{L}_{k}\right\}_{k \in \mathbb{Z}^{d}}$ of mutually disjoint measurable subsets of $\mathbb{T}^{d}$ such that $\cup_{k \in \mathbb{Z}^{d}}\left(k+\mathcal{L}_{k}\right)=\Omega$, where this equality is again meaningful only in a measure-theoretic sense, for if it is not true there exists a subset $\mathcal{U}$ of $\mathbb{T}^{d}$ with positive measure and $l_{1}, l_{2}$ in $\mathbb{Z}^{d}, l_{1} \neq l_{2}$, such that $\mathcal{U}+l_{1}$ and $\mathcal{U}+l_{2}$ are contained in $\Omega$. Then, the inverse Fourier transform $\left(\chi_{\mathcal{U}+l_{1}}\right)^{\vee}$ belongs to $V_{0}$, so

$$
\chi \mathcal{U}+l_{1}=m \hat{\omega}
$$

with $m \in L^{2}\left(\mathbb{T}^{d}\right)$. Since supp $\hat{\omega}=\Omega$ and $m$ is $\mathbb{Z}^{d}$-periodic, we conclude $m(\xi) \hat{\omega}(\xi) \neq 0$ a.e. on $\mathcal{U}+l_{2}$ which is disjoint from $\mathcal{U}+l_{1}$. This conclusion directly contradicts (3), so our claim is proved. 
Next, we claim that the previously established property of $\Omega$ combined with its radiality imply $\Omega \subseteq \mathbb{T}^{d}$. To prove this claim, assume that the contrary is true. Then, there exists a point of density $\xi_{0}$ of $\Omega$ and $p \in \mathbb{Z}^{+}$ such that $\xi_{0} \in\left(p \mathbf{i}+\mathbb{T}^{d}\right)$, where $\mathbf{i}=(1,0, \ldots, 0)$. Consequently, every point on $\left\|\xi_{0}\right\| \mathbb{S}^{d-1}$ is a density point of $\Omega$. Let, $C_{1}:=\left\|\xi_{0}\right\| \mathbb{S}^{d-1} \cap\left(p \mathbf{i}+\mathbb{T}^{d}\right) \neq \emptyset$ and $C_{2}:=\left\|\xi_{0}\right\| \mathbb{S}^{d-1} \cap\left(p \mathbf{j}+\mathbb{T}^{d}\right) \neq \emptyset$, where $\mathbf{j}=(0,1, \ldots, 0)$. Since every point in both $C_{1}$ and $C_{2}$ is a density point of $\Omega$, these two sets must be contained in $p \mathbf{i}+\mathcal{L}_{p \mathbf{i}}$ and $p \mathbf{j}+\mathcal{L}_{p \mathbf{j}}$ respectively, where $\mathcal{L}_{p \mathbf{i}}$ and $\mathcal{L}_{p \mathbf{j}}$ are disjoint. But, $-p \mathbf{i}+C_{1}$ and $-p \mathbf{j}+C_{2}$ intersect at a density point of both $\mathcal{L}_{p \mathbf{i}}$ and $\mathcal{L}_{p \mathbf{j}}$ contradicting the fact that these two sets are disjoint. Thus, $\Omega \subseteq \mathbb{T}^{d}$. The converse implication is obvious.

Remark 2.3. If $V=P W_{\Omega}$, for some radial subset $\Omega$ we can find a subset $F \subset L^{2}\left(\mathbb{R}^{d}\right)$ with $V=$ $\overline{\operatorname{span}}\left\{T_{n} f: f \in F, n \in \mathbb{Z}^{d}\right\}$ and this set is at most countably infinite. To show the existence of such a set it suffices to apply Zorn's lemma. The construction of the set of generator $F$ is non-trivial but it does not interest us here. If $\Omega$ is essentially bounded, then every $f$ in $F$ can be chosen to satisfy $\left\{\xi \in \mathbb{R}^{d}: \widehat{f}(\xi) \neq\right.$ $0\}=\Omega$ with $F$ finite.

Example 2.4. The sequence of closed subspaces $V_{j}=P W_{2^{j} B(0, \rho)}$, for any $\rho>0$ and $j \in \mathbb{Z}$ is an IMRA.

The purpose of the next two examples is to show that: First, $\Omega$ can be unbounded, and second, $\Omega$ may not contain a neighborhood of the origin. Notice that such a neighborhood is sufficient for Condition (ii). In both examples we use dyadic dilations.

Example 2.5. Denote by $B(0, r, s)$ the ( $d$-dimensional) spherical shell centered at the origin having inner radius $r$ and outer radius $s$. Now for simplicity set $d=2$ although this example can be generalized for every $d>2$. Let $\mathbf{A}=\bigcup_{n=1}^{\infty} B\left(0, r_{n}, 2^{n-1}\right)$, with $r_{n}=2^{n-1}-(1 / 16)^{n}$, and let $\mathbf{B}=B(0,1 / 2)$. Set $\Omega:=\mathbf{A} \cup \mathbf{B}$. Since $\mathbf{A}$ and $\mathbf{B}$ are disjoint, $|\mathbf{A} \cup \mathbf{B}|=|\mathbf{A}|+|\mathbf{B}|$. Moreover, both $\mathbf{A}$ and $\mathbf{B}$ have finite measure. Since $\left|B\left(0, r_{n}, 2^{n-1}\right)\right|=\pi\left[(1 / 8)^{n}-(1 / 16)^{2 n}\right]$ which implies $|\mathbf{A}|<\infty$. Thus, $\Omega$ is radial and satisfies all the conditions (i)-(iii) of Proposition 2.2: (i) is satisfied because $B\left(0, r_{n+1}, 2^{n}\right) \subset 2 B\left(0, r_{n}, 2^{n-1}\right)$ for all $n \geq 1$ and $B\left(0, r_{1}, 1\right) \subset 2 \mathbf{B}$; (ii) is trivially satisfied since $\mathbf{B}$ is contained in $\Omega$. The fact that $\Omega$ has finite measure implies (iii). Thus, $\Omega$ gives rise to an Isotropic Multiresolution Analysis, but $\Omega$ is not bounded.

Example 2.6. This example is valid for every $d \geq 2$. Let $\Omega=\bigcup_{n=1}^{\infty} B\left(0,(1 / 2)^{n+1}, r_{n}\right)$, with $r_{n}=(1 / 2)^{n}-$ $(1 / 100)^{n}$. Obviously, $\Omega$ contains no neighborhood of the origin. According to Proposition 2.2 and since, $\Omega \subseteq B(0,1 / 2)$ it is enough to prove that the complement of $\bigcup_{n=1}^{\infty} 2^{j} \Omega$ is null and $\Omega \subset 2 \Omega$ in order to establish that $\Omega$ defines an IMRA. Let us first show that (i) of Proposition 2.2 is satisfied. This property holds because $2 B\left(0,(1 / 2)^{n+1}, r_{n}\right)=B\left(0,(1 / 2)^{n}, 2 r_{n}\right)$ and $r_{n-1}<2 r_{n}$ for all $n \geq 2$.

We now prove that (ii) is satisfied. Let $x \in \mathbb{R}^{d}$ and $j_{x} \in \mathbb{Z}$ such that $2^{j_{x}-1}<\|x\|<2^{j_{x}}$. Then,

$$
\frac{1}{4}<\left\|\frac{x}{2^{j_{x}+1}}\right\|<\frac{1}{2}
$$

However,

$$
\lim _{n \rightarrow \infty} \frac{r_{n}-2^{-(n+1)}}{2^{-(n+1)}}=1,
$$

so there exists positive integer $n_{x}$ satisfying

$$
\frac{\left\|\frac{x}{2^{j x}+1}\right\|-\frac{1}{4}}{1 / 4}<\frac{r_{n_{x}}-2^{-\left(n_{x}+1\right)}}{2^{-\left(n_{x}+1\right)}} .
$$

This implies that $2^{-\left(j_{x}+1\right)} x$ belongs to the spherical shell $2^{n_{x}-1} B\left(0,2^{-\left(n_{x}+1\right)}, r_{n_{x}}\right)=B\left(0, \frac{1}{4}, 2^{n_{x}-1} r_{n_{x}}\right)$. Thus, $x$ belongs to $2^{n_{x}+j_{x}} \Omega$. Our claim now follows from the fact that $\cup_{j \in \mathbb{Z}} 2^{j} \mathbb{S}^{d-1}$ is null.

Remark 2.7. Theorem 2.1 can be restated in terms of the theory of Von Neumann Algebras: The joint commutant of $\{\mathcal{O}: O \in S O(d)\}$ and $\mathfrak{T}_{\mathbb{Z}^{d}}$, on one hand, and the joint commutant of $\{\mathcal{O}: O \in S O(d)\}$ and $\mathfrak{T}_{\mathbb{R}^{d}}$ on the other hand coincide. This follows from the fact that the orthogonal projections in these two 
joint commutants are exactly the same, as Theorem 2.1 suggests. Here, $\mathfrak{T}_{G}$ denotes the group of translations induced by a subgroup $G$ of $\mathbb{R}^{d}$.

So far, we have characterized the 'core' or zero-resolution subspaces $V_{0}$ of IMRAs. We also note that if these are singly generated then the choices for 'core' IMRA-subspaces are all of one kind: $P W_{\Omega}$ with $\Omega$ radial and inside the fundamental domain $\mathbb{T}^{d}$. In the light of this discussion an interesting open problem is to characterize all sets $\Omega$ for which $V_{0}=P W_{\Omega}$ is generated by a fixed number, $N$, of functions. However, none of these generators can be compactly supported in the spatial domain: If one of them, say $\phi_{1}$, is compactly supported then $\widehat{\phi_{1}}$ has analytic extension on the complex domain $\mathbb{C}^{d}$. Then $\widehat{\phi_{1}}$ can vanish only on a set of points with no accumulation point, forcing $\Omega=\mathbb{R}^{d}$ which, in turn, implies $V_{0}=L^{2}\left(\mathbb{R}^{d}\right)$ which contradicts the IMRA-definition.

\section{ISOTROPIC REFINABLE FUNCTIONS AND IMRAS}

According to Proposition 2.2 a single refinable function $\phi$ defines an IMRA by letting $V_{0}=\overline{\operatorname{span}}\left\{T_{n} \phi\right.$ : $\left.n \in \mathbb{Z}^{d}\right\}$, if $\phi$ is radial and the support of $\hat{\phi}$ is contained in the fundamental domain $\mathbb{T}^{d}$. This observation motivates us to characterize radial and, more generally, isotropic refinable functions (Theorem 3.1) with respect to dilations defined by radially expansive matrices. The purpose of this section is to characterize these functions. As we will see not all of these functions generate 'core' IMRA-subspaces, because their support in the frequency domain does not have to be contained in the fundamental domain. We begin with dyadic dilations in order to make our arguments more clear.

Let $A$ be a radially expansive matrix. With respect to dilations induced by $A$, a function $\phi$ in $L^{2}\left(\mathbb{R}^{d}\right)$ is called refinable if there exists a measurable, essentially bounded, $\mathbb{Z}^{d}$-periodic function $H$ such that $\widehat{\phi}\left(A^{*} \xi\right)=H(\xi) \widehat{\phi}(\xi)$, a.e. $\xi \in \mathbb{R}^{d}$. The function $H$ is called the low-pass filter or mask corresponding to $\phi$.

Theorem 3.1. Let $A$ be a radially expansive matrix and $\phi \in L^{2}\left(\mathbb{R}^{d}\right)$ be a refinable function and $H \in$ $L^{\infty}\left(\mathbb{T}^{d}\right)$ be its mask. We also assume that

(1) $\phi$ is isotropic, and

(2) $\lim _{\xi \rightarrow 0} \widehat{\phi}(\xi)=L \neq 0$.

Then $\phi \in P W_{\rho /(\rho+1)}$, where $\rho=|\operatorname{det} A|^{1 / d}$ is the dilation factor of $A$.

For the proof of this theorem we need the next lemma:

Lemma 3.2. The following holds:

$$
\operatorname{dist}\left(r \cdot \mathbb{S}^{d-1}, \mathbb{Z}^{d}\right)=\inf \left\{\|c-n\|: c \in r \cdot \mathbb{S}^{d-1}, n \in \mathbb{Z}^{d}\right\} .
$$

Then, $\lim _{r \rightarrow \infty} \operatorname{dist}\left(r \cdot \mathbb{S}^{d-1}, \mathbb{Z}^{d}\right)=0$.

Proof. It suffices to consider only the case $d=2$, since the projection of a $d$-sphere centered at the origin onto the $x_{1} x_{2}$-plane is a circle centered at the origin (with the same radius), and the projection of the lattice $\mathbb{Z}^{d}$ onto the $x_{1} x_{2}$-plane is $\mathbb{Z}^{2}$.

Pick $\epsilon>0$. Let $0<y_{0}<1$ be a value of the function $f_{1}(t):=\sqrt{1-t^{2}}$ with $0 \leq t \leq 1$ at $t=\tau_{0}$ satisfying $\left|f_{1}^{\prime}\left(\tau_{0}\right)\right|<\epsilon$. Observe that as $\epsilon$ the value $y_{0}$ tends to 1 . Now let $R_{\epsilon}>0$ satisfying $R_{\epsilon}\left(1-y_{0}\right)>2$. Then for every $R>R_{\epsilon}$ there is an integer $n_{0}$ between $R y_{0}$ and $R$. The graph of the function $f_{R}(t)=$ $\sqrt{R^{2}-t^{2}}, 0 \leq t \leq R$, intersects the horizontal line $y=n_{0}$ at some point $\left(t_{0}, n_{0}\right), t_{0}>0$. But $f_{R}$ is decreasing and $R y_{0} \leq n_{0} \leq R$. Thus, for all $0 \leq t \leq t_{0}$, we have $\left|f_{R}^{\prime}(t)\right|<\epsilon$. Since, the tangent line of the graph of $f_{R}(t)$ at the point $t=t_{0}$ intersects the vertical line $t=\left[t_{0}\right]$, where $\left[t_{0}\right]$ denotes the greatest integer not exceeding $t_{0}$, at a point whose $y$-coordinate belongs to the interval $\left[n_{0}, n_{0}+\left|f_{R}^{\prime}\left(t_{0}\right)\right|\left(t_{0}-\left[t_{0}\right]\right)\right]$. This implies that the distance between $\left(\left[t_{0}\right], n_{0}\right)$ and $R \mathbb{S}^{d-1}$ is less than $\epsilon$ (see Figure 2 ). 


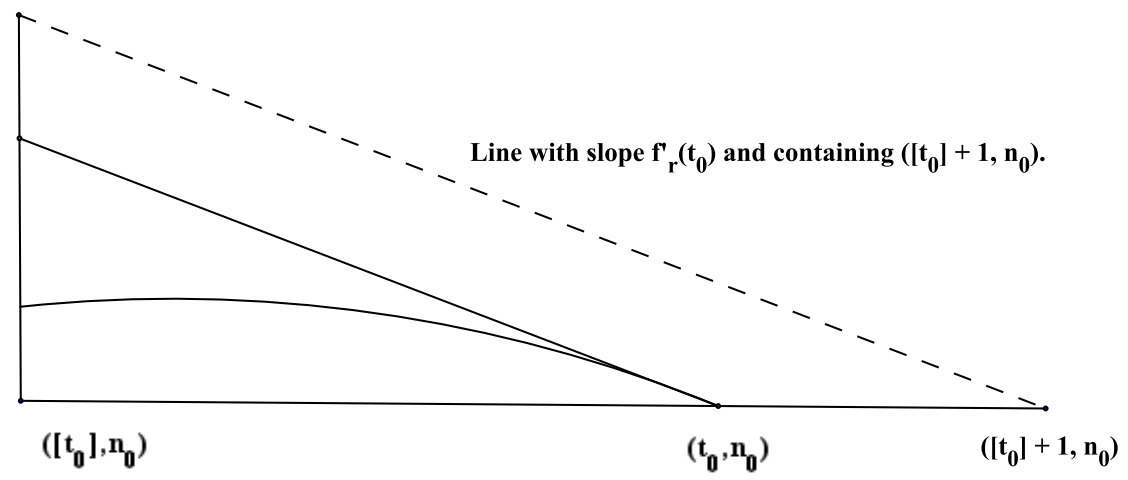

FIGURE 2. The dotted line is the line containing $\left(\left[t_{0}\right]+1, n_{0}\right)$ parallel to the tangent line to $R \cdot \mathbb{S}^{1}$ at $\left(t_{0}, n_{0}\right)$. The distance between the intersection point of the dotted line with the vertical line $x=\left[t_{0}\right]$ and the point $\left(\left[t_{0}\right], n_{0}\right)$ is precisely $\left|f_{R}^{\prime}\left(t_{0}\right)\right|$, thus the distance between $R \cdot \mathbb{S}^{1}$ and $\left(\left[t_{0}\right], n_{0}\right)$ is less than $\epsilon$.

The proof of the Theorem 3.1 contains technicalities which obscure some interesting geometric properties of $\phi$ and $H$ (which facilitate the proof). The strategy of the proof is to show that $|\hat{\phi}|$ is constant a.e. and thus $\phi$ cannot be square-integrable. To help the reader appreciate the underlying geometry, we begin by showing that the theorem is true when $\hat{\phi}$ is continuous.

Proof of Theorem 3.1. Without any loss of generality we may assume $\phi$ is radial, because the modulus of an isotropic refinable function is a radial refinable function. Set $\mathcal{N}=\left\{\xi \in \mathbb{R}^{d}: \widehat{\phi}(\xi)=0\right\}$.

Part 1: The first objective is to show that $\hat{\phi}$ has compact support. To this end, assume $\mathcal{N}^{c}$ is unbounded. Now, pick an arbitrary $\epsilon>0$.

Case I. Assume $\hat{\phi}$ is continuous. Since $\hat{\phi}$ is continuous, so is $\hat{\phi}\left(A^{*}\right.$. $)$. But, $\hat{\phi}(0)=L \neq 0$. Then, its mask $H \in L^{\infty}$ defined by the two-scale relation

$$
\hat{\phi}\left(A^{*} \xi\right)=H(\xi) \hat{\phi}(\xi) \quad \text { a.e. }
$$

can be extended to a continuous function on the open set $\mathcal{N}^{c}$ and, in particular, in a neighborhood of the origin, so that the previous equation hold for every $\xi \in \mathcal{N}^{c}$ and $H(0)=1$. We conclude, that there exists $\delta>0$ such that $|H(\xi)-1|<\epsilon$ for all $|\xi|<\delta$.

Notice that both $\mathcal{N}$ and $\mathcal{N}^{c}$ are radial. The previous lemma implies that there exists $R_{0}>0$ such that for all $R>R_{0}$ we have $\operatorname{dist}\left(R \mathbb{S}^{d-1}, \mathbb{Z}^{d}\right)<\delta / 2$. Since, $\mathcal{N}^{c}$ is not bounded, we can find a sphere with radius $R>R_{0}$ contained in $\mathcal{N}^{c}$. If $n \in \mathbb{Z}^{d}$ is a point of the integer grid satisfying $\operatorname{dist}\left(R \mathbb{S}^{d-1}, n\right)<\delta / 2$, then the ball $B(n, \delta)$ intersects the sphere $R \mathbb{S}^{d-1}$. The $\mathbb{Z}^{d}$-periodicity and the continuity of $H$ on the ball $B(0, \delta)$ now imply that for all $\xi \in B(n, \delta) \cap R \mathbb{S}^{d-1}$ we have $1-\epsilon<|H(\xi)|<1+\epsilon$. Since, all these $\xi$ belong to $\mathcal{N}^{c}$ the two-scale relation (4) gives

$$
1-\epsilon<\left|\frac{\hat{\phi}\left(A^{*} \xi\right)}{\hat{\phi}(\xi)}\right|<1+\epsilon \quad \text { for all } \xi \in R \mathbb{S}^{d-1},
$$

which in turn, implies $1-\epsilon<|H(\xi)|<1+\epsilon$ for every point on $R \mathbb{S}^{d-1}$. 
Next we show $|H|=1$ a.e. on $B(0,1 / 2)$. The key to this result is to be able to form the quotient $\hat{\phi}\left(A^{*}.\right) / \hat{\phi}$. We observe that all the points in the ball $B(0,1 / 2)$ satisfy the following: First, the denominator $\hat{\phi}(\xi)$ is non-zero; second, both $\hat{\phi}\left(A^{*} \xi\right)$ and $\hat{\phi}(\xi)$ are zero; and third $\hat{\phi}\left(A^{*} \xi\right) \neq 0$ and $\hat{\phi}(\xi)=0$. The two-scale relation (4) rules out the third case. For all points falling in the first case the quotient $\hat{\phi}\left(A^{*} \xi\right) / \hat{\phi}(\xi)=H(\xi)$, while in the second we can set $H$ equal to 1, because the two-scale relation (4) is satisfied.

We want to prove

$$
1-\epsilon<\left|\hat{\phi}\left(A^{*} \xi\right) / \hat{\phi}(\xi)\right|<1+\epsilon \quad \text { for all } \xi \in B(0,1 / 2) \cap \mathcal{N}^{c} .
$$

Let, $\xi_{0} \in B(0,1 / 2) \cap \mathcal{N}^{c}$ with $\left\|\xi_{0}\right\|>\delta$. We choose to such $\xi_{0}$ because all points inside the ball $B(0, \delta)$ satisfy (5). The radiality of $\mathcal{N}^{c}$ implies that the sphere $\left\|\xi_{0}\right\| \mathbb{S}^{d-1}$ is contained in $\mathcal{N}^{c}$. Thus,

$$
H(\xi)=\hat{\phi}\left(A^{*} \xi\right) / \hat{\phi}(\xi) \quad \text { for every } \xi \in\left\|\xi_{0}\right\| \mathbb{S}^{d-1} \text {. }
$$

Since $H$ is $\mathbb{Z}^{d}$-periodic and the sphere $n+\left\|\xi_{0}\right\| \mathbb{S}^{d-1}$ intersects $R \mathbb{S}^{d-1}$, we derive that (5) is valid for every point of $\left\|\xi_{0}\right\| \mathbb{S}^{d-1}$. Since for all other points in $B(0,1 / 2)$ we have $|H|=1$, we conclude $1-\epsilon<|H|<1+\epsilon$ everywhere on $B(0,1 / 2)$. Since $\epsilon$ is arbitrary, we conclude $|H|=1$ on $B(0,1 / 2)$. This in turn gives that $|\hat{\phi}|$ is constant on $B(0,1 / 2)$.

Next take the radial profile of $\hat{\phi}$ on one of the Cartesian coordinate axes, say the one parallel to the vector $\mathbf{i}=(1,0, \ldots, 0)$. The periodicity of $H$ implies that $|H|$ is constant throughout this axis. This fact combined with the (4) imply that $|\hat{\phi}|$ is constant on the intervals $\left[2^{j-1}, 2^{j}\right)$ for all $j=1,2, \ldots$ Thus $|\hat{\phi}|$ is constant on the coordinate axis parallel to $i$. This fact combined with the radiality of $|\hat{\phi}|$ imply that $|\hat{\phi}|$ is constant on its radial profile, so $|\hat{\phi}|$ is constant, which contradicts that $\phi \in L^{2}\left(\mathbb{R}^{d}\right)$.

Case II. Remove the continuity assumption for $\hat{\phi}$. As in Case I, let $\delta>0$ such that $|H(\xi)-1|<\epsilon$ for all $|\xi|<\delta$. Obviously, the same property implies that $H$ can be redefined, if necessary, so that $H(0)=1$ and $H$ is continuous at the origin. The difficulty in this more general case comes from the fact that spheres are sets of zero measure so they do not determine $\hat{\phi}$. This forces us to use spherical shells instead of spheres. However, these spherical shells may contain points from both $\mathcal{N}$ and its complement, a situation not possible in the previous case: Spheres due to the radiality of $\hat{\phi}$ contain points only of one of these two sets.

Lemma 3.2 gives $R_{0}>0$ such that for all $R>R_{0}$ we have $\operatorname{dist}\left(r \mathbb{S}^{d-1}, \mathbb{Z}^{d}\right)<\delta / 4$. Since, $\mathcal{N}^{c}$ is not bounded, we can find a spherical shell centered at the origin with inner radius $R_{1}$ and outer radius $R_{2}$ denoted by $B\left(0, R_{1}, R_{2}\right)$, so that $R_{2}-R_{1}<\delta / 2$ and $\left|\mathcal{N}^{c} \cap B\left(0, R_{1}, R_{2}\right)\right|>0$. In fact, we can select $R_{1}>R_{0}$. Then, $\operatorname{dist}\left(\frac{R_{1}+R_{2}}{2} \mathbb{S}^{d-1}, \mathbb{Z}^{d}\right)<\delta / 4$, so let $n_{1} \in \mathbb{Z}^{d}$ to satisfy,

$$
\operatorname{dist}\left(\frac{R_{1}+R_{2}}{2} \mathbb{S}^{d-1}, n_{1}\right)<\delta / 4 \text {. }
$$

Now notice that the selection of the radii of the spherical shell implies $R_{1}<\left\|n_{1}\right\|<R_{2}$. Thus the interval $\left(\left\|n_{1}\right\|-\delta,\left\|n_{1}\right\|+\delta\right)$ contains a radial profile of $\mathcal{N}^{c} \cap B\left(0, R_{1}, R_{2}\right)$. Moreover, the intersection of $B\left(n_{1}, \delta\right)$ and $\mathcal{N}^{c} \cap B\left(0, R_{1}, R_{2}\right)$ has positive measure, and for a.e. $\xi$ in this intersection the 2-scale relation $\hat{\phi}\left(A^{*} \xi\right)=H(\xi) \hat{\phi}(\xi)$ holds. Combining this fact with the radiality of $\hat{\phi}$, and thus, of $\hat{\phi}\left(A^{*} \cdot\right)$, we infer

$$
1-\epsilon<\left|\frac{\hat{\phi}\left(A^{*} \xi\right)}{\hat{\phi}(\xi)}\right|<1+\epsilon \quad \text { for a.e. } \xi \in B\left(0, R_{1}, R_{2}\right) \cap \mathcal{N}^{c} .
$$

This shows

$$
1-\epsilon<|H(\xi)|<1+\epsilon \quad \text { for a.e. } \xi \in B\left(0, R_{1}, R_{2}\right) \cap \mathcal{N}^{c} .
$$

Continuing towards our objective we must show $|H|=1$ a.e. on $B(0,1 / 2)$. Arguing as in Case I we have that for almost all points in $B(0,1 / 2) \cap \mathcal{N}^{c}$ we have $H(\xi)=\hat{\phi}\left(A^{*} \xi\right) / \hat{\phi}(\xi)$, while if $\hat{\phi}\left(A^{*} \xi\right)=\hat{\phi}(\xi)=0$ we set $H(\xi)=1$. 
Similarly to Case I, but now without the benefit of the continuity assumption of $\hat{\phi}$ we want to establish

$$
1-\epsilon<\left|\hat{\phi}\left(A^{*} \xi\right) / \hat{\phi}(\xi)\right|<1+\epsilon \quad \text { for a.e. } \xi \in B(0,1 / 2) \cap \mathcal{N}^{c} \text {. }
$$

Take a density point of $B(0,1 / 2) \cap \mathcal{N}^{c}$, say $\xi_{0}$ with $\left\|\xi_{0}\right\|>\delta$, since points inside the ball $B(0, \delta)$ satisfy (7). The radiality of $\mathcal{N}^{c}$ implies that all points on the sphere $\left\|\xi_{0}\right\| \mathbb{S}^{d-1}$ are density points of $\mathcal{N}^{c}$. Since $\left\|\xi_{0}\right\|>\delta$, the sphere $n_{1}+\left\|\xi_{0}\right\| \mathbb{S}^{d-1}$ intersects $B\left(0, R_{1}, R_{2}\right)$ from the inner boundary throughout its outer boundary and the radiality of $\mathcal{N}^{c}$ implies that a density point of $\mathcal{N}^{c} \cap B\left(0, R_{1}, R_{2}\right)$, say $\xi_{1}$, belongs to $n_{1}+\left\|\xi_{0}\right\| \mathbb{S}^{d-1}$. Consequently,

$$
\lim _{\eta \rightarrow 0} \frac{\left|B\left(\xi_{1}, \eta\right) \cap \mathcal{N}^{c} \cap B\left(0, R_{1}, R_{2}\right)\right|}{\left|B\left(\xi_{1}, \eta\right)\right|}=1
$$

which combined with the periodicity of $H$ and (6) imply that $-n_{1}+\xi_{1}$ which belongs to $\left\|\xi_{0}\right\| \mathbb{S}^{d-1}$ is a density point of the set $\{\xi \in B(0,1 / 2): 1-\epsilon<|H(\xi)|<1+\epsilon\}$ is satisfied. We have now established that a.e. in $B(0,1 / 2)$ we have $1-\epsilon<|H|<1+\epsilon$. Since $\epsilon$ is arbitrary we conclude $|H|=1$ on $B(0,1 / 2)$. This implies that $|\hat{\phi}|$ is a.e. constant on $B(0,1 / 2)$ and due to the two-scale relation this result extends to the ball $B(0, \rho / 2)$.

The final step of the first part of the the proof is to show that the latter result is true for all spherical shells $B\left(0, \rho^{l} / 2, \rho^{l+1} / 2\right)$ with $l=1,2,3, \ldots$ Which gives $|\hat{\phi}|$ is constant a.e. on $\mathbb{R}^{d}$ and thus it cannot be square-summable, and hence $\hat{\phi}$ has compact support. We examine the case $l=1$ only since the arguments in this case generalize immediately for every $l>1$.

To establish that $|\hat{\phi}|$ is a.e. constant equal to $|L|$ on $B\left(0, \rho^{l} / 2, \rho^{l+1} / 2\right)$, where $l=1,2,3, \ldots$, it is enough to consider the case $l=1$, since all other cases are identical. Recall that, for continuous $\hat{\phi}$ we used the fact that $|H|=1$ on every coordinate axis. However, those axes are null sets so this argument needs to be appropriately modified when this hypothesis is no longer valid.

Suppose that $\Theta$ be a set of positive measure on the unit sphere and $B\left(0, R_{1}, R_{2}, \Theta\right):=\left\{(r, \theta): R_{1}<\right.$ $\left.r<R_{2}, \quad \theta \in \Theta\right\}$. Let $\Theta_{n}$ be a sequence of open neighborhoods of $\left(\rho^{2} / 2\right) \mathbf{i}$ such that $m\left(\Theta_{n}\right) \downarrow 0$, where $n=1,2, \ldots$. Define

$$
L_{n}:=\bigcup_{k \in \mathbb{Z}^{+}}\left(k+B\left(0, \frac{1}{2}\right)\right) \cap B\left(0, \frac{1}{2}, \frac{\rho}{2}, \Theta_{n}\right) .
$$

Pick $\rho / 2<r<\rho^{2} / 2$. Then there exists a sufficiently large $n$ so $L_{n}$. contains a ball $B\left(\frac{r}{\rho} \mathbf{i}, \eta\right)$. Using the periodicity of $H$ we have that $|H|=1$ on every $L_{n}$. Combining the two-scale relation (4) with the facts that $|\hat{\phi}|$ is constant on the ball $B\left(0, \frac{\rho}{2}\right)$ and $|H|=1$ on every $L_{n}$, we conclude

$$
|L|=|\hat{\phi}|_{B(r \mathbf{i}, \rho \eta)} \mid \text {. }
$$

Therefore, the point $r \mathbf{i}$ is a density point of the set $\left\{\xi \in \mathbb{R}^{d}:|\hat{\phi}(\xi)|=|L|\right\}$, which is radial since $\hat{\phi}$ is radial. In conclusion, $|\hat{\phi}(\xi)|=|L|$ a.e. on the spherical shell $B\left(0, \rho / 2, \rho^{2} / 2\right)$.

Part 2: This part establishes that the support of $\hat{\phi}$ is contained in the ball $B\left(0, \frac{\rho}{\rho+1}\right)$. Since $\hat{\phi}$ has compact support, we can find

$$
R=\inf \left\{r>0: \mathcal{N}^{c} \subseteq B(0, r)\right\} .
$$

Therefore, we can find spheres centered at the origin with radii arbitrarily close to $R$ consisting of density points of $\mathcal{N}^{c}$. First, assume $R>1$. Pick a sufficiently small $\delta>0$ and one such sphere, say $B\left(0, R_{0}\right)$ with $R_{0}>R-\delta>1$. Eq. (4) rewritten as

$$
\hat{\phi}(\xi)=H\left(\left(A^{*}\right)^{-1} \xi\right) \hat{\phi}\left(\left(A^{*}\right)^{-1} \xi\right) \quad \text { a.e. }
$$

and the radiality of $\mathcal{N}^{c}$ imply that every point on the sphere $B\left(0, R_{0} / \rho\right)$ is a density point for $\mathcal{N}^{c}$ and a density point of the set $\mathcal{S}:=\left\{\xi \in \mathbb{R}^{d}: H(\xi) \neq 0\right\}$. If $\kappa$ is the greatest integer not exceeding $R_{0}$, then the spheres $R_{0} \mathbb{S}^{d-1}$ and $(\kappa-1) \mathbf{i}+\left(R_{0} / \rho\right) \mathbb{S}^{d-1}$ intersect. The periodicity of $H$ implies that every point on $\kappa+\left(R_{0} / \rho\right) \mathbb{S}^{d-1}$ is a density point of $\mathcal{S}$. By observing that the distance of the centers of these two spheres 
is equal to $\kappa-1$ we infer that these spheres intersect. The common points of the two spheres are points of density for both $\mathcal{N}^{c}$ and $\mathcal{S}$ and, by the two-scale relation (4) all them are density points of the support of $\hat{\phi}\left(A^{*}\right.$.), which is absurd, because this function is supported on the ball $B(0, R / \rho)$. Therefore, $R \leq 1$. Take again $R_{0}$ as above but this time we have $R-\delta<R_{0}<1$. The spheres $R_{0} \mathbb{S}^{d-1}$ and $\mathbf{i}+\left(R_{0} / \rho\right) \mathbb{S}^{d-1}$ intersect unless $R_{0} \leq \frac{\rho}{\rho+1}$. Since $\delta$ can be arbitrarily small we conclude $R \leq \frac{\rho}{\rho+1}$.

Theorem 3.1 has two important ramifications.

Corollary 3.3. There is no isotropic, refinable function compactly supported in the spatial domain.

According to Proposition 2.2 the refinable functions generating an integer shift and rotation-invariant subspace of $L^{2}\left(\mathbb{R}^{d}\right)$ belong to the Paley-Wiener space $P W_{\mathbb{T}^{d}}$. So if a refinable function $\phi$ is isotropic and does not belong to $P W_{\mathbb{T}^{d}}$, does it define an IMRA? The answer is provided by the next corollary.

Corollary 3.4. If an MRA is defined by a single isotropic refinable function $\phi$ whose Fourier transform is supported outside the ball of radius $1 / 2$ centered at the origin is not an IMRA; equivalently, the orthogonal projection onto $V_{0}$ does not commute with all rotations.

We remark that Theorem 3.1 cannot be extended for non-singleton refinable sets $\left\{\phi_{l}: l \in \mathbb{N}\right\}$, in the sense that there exists an essentially bounded $\infty \times \infty$-matrix valued $\mathbb{Z}^{d}$-periodic function $H$ satisfying

$$
\left(\hat{\phi}_{1}\left(A^{*} \cdot\right), \hat{\phi}_{2}\left(A^{*} \cdot\right), \ldots\right)^{T}=H\left(\hat{\phi}_{1}, \hat{\phi}_{2}, \ldots\right)^{T} \quad \text { a.e. on } \mathbb{R}^{d},
$$

where all $\phi_{l}$ are isotropic. An example of such a set can be manufactured from Example 2.5.

3.1. Isotropic wavelets for refinable functions. The goal of this subsection is to provide an easy method for constructing isotropic wavelets from refinable radial functions. This method can be applied to singly generated IMRAs as well as to refinable functions $\phi$ for which $\hat{\phi}$ not supported in the fundamental domain $\mathbb{T}^{d}$ and which are not associated with singly generated IMRAs. Since the primary focus of this paper is this type of IMRAs we will not study the latter type of refinable functions in detail. We consider radial refinable with respect to a radially expansive matrix $A$ functions, whose Fourier transforms do not vanish in the ball of radius $\rho /(\rho+1)$, where $\rho=|\operatorname{det} A|$. However, the gains from using these functions are limited thanks to technicalities which will become apparent in the following discussion. To allow some notational simplicity we limit this discussion to the case $A=2 I_{d}$ and to $d=2$. Then, by virtue of Theorem (3.1), the support of $\hat{\phi}$ is the ball $B(0,2 / 3)$. The simplest case, is for $\hat{\phi}=\chi_{B(0,2 / 3)}$.

Take $I$ to be the $d$-dimensional spherical shell with outer radius $4 / 3$ and inner radius $2 / 3$ and $\epsilon_{l}(\xi):=$ $e^{2 \pi i\left[\frac{l}{3} \cdot \xi\right]}$, where $\xi \in \mathbb{R}^{2}$. Define $\widehat{\psi}=\chi_{I}$. The spherical shell $I$ is contained in the square $[-3 / 2,3 / 2]^{2}$. Since, the set $\left\{\frac{1}{3} \epsilon_{l}: l \in \mathbb{Z}^{2}\right\}$ is an orthonormal basis for $L^{2}\left([-3 / 2,3 / 2]^{2}\right)$ we deduce that $\left\{\frac{1}{3} \epsilon_{l} \widehat{\psi}: l \in \mathbb{Z}^{2}\right\}$ is a Parseval frame for $P W_{I}$. Taking the residue classes of the integers modulo 3 we can rewrite this set in the following way

$$
\left\{e_{n}\left(\frac{1}{3} \epsilon_{(\mu, \nu)} \widehat{\psi}\right): \mu, \nu=0,1,2, n \in \mathbb{Z}^{2}\right\} .
$$

After, setting $\psi_{(\mu, \nu)}$ to be the inverse Fourier transform of $\frac{1}{3} \epsilon_{(\mu, \nu)} \widehat{\psi}$ we conclude that

$$
\left\{T_{n} \psi_{(\mu, \nu)}: n \in \mathbb{Z}^{2}, \mu, \nu=0,1,2\right\}
$$

is a Parseval frame for $P W_{I}$. By applying $D_{2 I_{2}}^{j}$ to the previous Parseval frame we derive a Parseval frame for each $P W_{2^{j} I}$ for every $j \in \mathbb{Z}$. Therefore,

$$
\left\{D_{2 I_{2}}^{j} T_{n} \psi_{(\mu, \nu)}: n \in \mathbb{Z}^{2}, \mu, \nu=0,1,2, j \in \mathbb{Z}\right\}
$$

is a Parseval frame for $L^{2}\left(\mathbb{R}^{2}\right)$. It's worth noting that $P W_{I}$ is not the relative orthocomplement of the Fourier transform of $V_{0}$ inside the Fourier transform of $V_{1}$; for otherwise $V_{0}$ would have been a Paley-Wiener space.

The next step is to extend this construction of isotropic wavelets for the case of a refinable function $\phi$ whose Fourier transform is continuous and does not vanish in the ball $B(0,2 / 3)$. This construction is easily generalizable for radii other not exceeding $2 / 3$. Take a function $\vartheta \in L^{2}(\mathbb{R})$ such that 
- $\vartheta \in P W_{[-4 / 3,4 / 3]}$,

- $\widehat{\vartheta} \geq 0$, and even

- $\widehat{\vartheta}$ is strictly positive on some annulus $B(0,4 / 3) \backslash B\left(0, b_{2}\right)$, for some $b_{2}<\frac{2}{3}$,

- $\widehat{\vartheta}$ is zero on a neighborhood of the origin contained in $\left(-\frac{2}{3}, \frac{2}{3}\right)$,

- $\sum_{j \in \mathbb{Z}}\left|\widehat{\vartheta}\left(2^{j} \xi\right)\right|^{2}=1$ a.e. $\xi \in \mathbb{R}$.

Let $\psi \in P W_{B(0,4 / 3)}$ be defined by $\widehat{\psi}(\xi)=\widehat{\vartheta}(\|\xi\|)$, where $\xi \in \mathbb{R}^{2}$ and $(\mu, \nu) \in\{0,1,2\} \times\{0,1,2\}$

$$
\widehat{\psi_{(\mu, \nu)}}(\xi)=\frac{1}{3} \epsilon_{(\mu, \nu)}(\xi) \widehat{\psi}(\xi) \text {. }
$$

The arguments we previously used no longer apply, since multiplication by $\hat{\psi}$ no longer defines a projection. So to conclude that

$$
\left\{D_{2 I_{2}}^{j} T_{n} \psi_{(\mu, \nu)}: n \in \mathbb{Z}^{2}, \mu, \nu=0,1,2, j \in \mathbb{Z}\right\}
$$

is a Parseval frame of $L^{2}\left(\mathbb{R}^{2}\right)$ we must use a different method. By means of a characterization of affine Parseval frames due to Han [41] (for $d=1$ the same result is due to Wang [44]) we have to show

$$
\left.\sum_{\mu, \nu=0}^{2} \sum_{j \in \mathbb{Z}} \widehat{\mid \psi_{(\mu, \nu)}}\left(2^{j} \xi\right)\right|^{2}=1 \text { a.e. } \xi \in \mathbb{R}^{2},
$$

$$
\sum_{\mu, \nu=0}^{2} \sum_{j=0}^{\infty} \widehat{\psi_{(\mu, \nu)}}\left(2^{j} \xi\right) \overline{\widehat{\psi_{(\mu, \nu)}}\left(2^{j}(\xi+s)\right)}=0, \quad \text { a.e. } \xi \in \mathbb{R}^{2} \text { and all } s \in \mathbb{Z}^{2} \backslash 2 \mathbb{Z}^{2}
$$

in order to establish that $\left\{D_{2 I_{2}}^{j} T_{n} \psi_{(\mu, \nu)}: n \in \mathbb{Z}^{2}, \mu, \nu=0,1,2, j \in \mathbb{Z}\right\}$ is a Parseval frame of $L^{2}\left(\mathbb{R}^{2}\right)$.

The last property in the definition of $\hat{\psi}$ immediately implies

$$
\sum_{\mu, \nu=0}^{2} \sum_{j \in \mathbb{Z}}\left|\widehat{\psi(\mu, \nu)}\left(2^{j} \xi\right)\right|^{2}=1 \text { a.e. } \xi \in \mathbb{R}^{2} .
$$

To establish that (8) holds for the set of isotropic wavelets $\left\{\psi_{(\mu, \nu)}: \mu, \nu=0,1,2\right\}$ it suffices to consider the cases $j=0$ and $j=1$, because the supports of $\widehat{\psi}\left(2^{j} \cdot\right)$ and $\widehat{\psi}\left(2^{j}(\cdot+s)\right)$ are disjoint, if $j>1$. When $j=0$ and $j=1$ the summands in the right-hand side of (8) are of the form

$$
\hat{\psi}\left(2^{j} \xi\right) \overline{\hat{\psi}\left(2^{j}(\xi+s)\right)} \sum_{\mu=0}^{2} \sum_{\nu=0}^{2} e^{-2 \pi i \frac{\mu}{3} 2^{j} s_{1}} e^{-2 \pi i \frac{\nu}{3} 2^{j} s_{2}},
$$

where $s=\left(s_{1}, s_{2}\right)$. When, $j=0$ the supports of $\widehat{\psi}\left(2^{j} \cdot\right)$ and $\widehat{\psi}\left(2^{j}(\cdot+s)\right)$ are balls with radius $4 / 3$ centered at the origin and at $-s=-\left(s_{1}, s_{2}\right)$ respectively. Therefore, if $\left|s_{1}\right|>2$ or $\left|s_{2}\right|>2$ hold then, the supports of $\widehat{\psi}\left(2^{j} \cdot\right)$ and $\widehat{\psi}\left(2^{j}(\cdot+s)\right)$ are again disjoint. Thus the only allowable values for $s_{1}$ and $s_{2}$ are $0, \pm 1, \pm 2$. Moreover, the requirement $s \in \mathbb{Z}^{2} \backslash 2 \mathbb{Z}^{2}$ rules out the cases $\left|s_{1}\right|=\left|s_{2}\right|=2$ and $s_{1}=s_{2}=0$. For all other choices of $s_{1}$ and $s_{2}$, first note

$$
\sum_{\mu=0}^{2} \sum_{\nu=0}^{2} e^{-2 \pi i \frac{\mu}{3} s_{1}} e^{-2 \pi i \frac{\nu}{3} s_{2}}=\left(\sum_{\mu=0}^{2} e^{-2 \pi i \frac{\mu}{3} s_{1}}\right)\left(\sum_{\nu=0}^{2} e^{-2 \pi i \frac{\nu}{3} s_{2}}\right) .
$$

Since, for these choices of $s_{1}$ and $s_{2}$ at least one of $e^{-2 \pi i \frac{s_{1}}{3}}$ and $e^{-2 \pi i \frac{s_{2}}{3}}$ is a generator of the cyclic multiplicative group of the third-order roots of unity, we infer that at least one of the factors in the right-hand side of the previous equation is equal to zero. 
When $j=1$, similar arguments show that $s_{1}$ and $s_{2}$ belong to $\{0, \pm 1\}$ with the exception that both $s_{1}$ and $s_{2}$ may not be equal to zero. This time we have

$$
\sum_{\mu=0}^{2} \sum_{\nu=0}^{2} e^{-4 \pi i \frac{\mu}{3} s_{1}} e^{-4 \pi i \frac{\nu}{3} s_{2}}=\left(\sum_{\mu=0}^{2} e^{-4 \pi i \frac{\mu}{3} s_{1}}\right)\left(\sum_{\nu=0}^{2} e^{-4 \pi i \frac{\nu}{3} s_{2}}\right) .
$$

Observing once again that at least one of $e^{-2 \pi i \frac{2 s_{1}}{3}}$ and $e^{-2 \pi i \frac{2 s_{2}}{3}}$ is a generator of the cyclic multiplicative group of the third-order roots of unity, we obtain the desired result. This completes the proof that the $\psi_{\mu, \nu}$ 's satisfy (8) for every $s \in \mathbb{Z}^{2} \backslash 2 \mathbb{Z}^{2}$.

Remark 3.5. 1) When the support of the Fourier transform of the radial refinable function $\phi$ is contained in $\mathbb{T}^{d}$, then we can use fewer modulation factors to define the associated isotropic wavelets. For example, if $d=2$ and $A=D_{2 I_{2}}$, instead of the modulation factors $\frac{1}{3} \epsilon_{(\mu, \nu)}$ we can use $\varepsilon_{(\mu, \nu)}$, where $\mu, \nu \in\{0,1\}$ and $\varepsilon_{(\mu, \nu)}(\xi):=e^{\pi i[(\mu, \nu) \cdot \xi]}$. The use of refinable functions that do not define singly generated IMRAs forces us to use more modulations to define the isotropic wavelets that form a Parseval frame of $L^{2}\left(\mathbb{R}^{d}\right)$. The cost of using such refinable functions whose support in the frequency domain is not significantly 'bigger' than the ball $B(0,1 / 2)$ does not offset the gains. Therefore, we are justified to restrict our attention to refinable isotropic functions which generate IMRAs.

2) The method presented in this subsection for construction of isotropic wavelets associated with radial refinable functions does not show how to implement fast algorithms based on IMRAs for the multiscale decomposition and reconstruction of multidimensional data sets. This observation motivates the next section.

\section{EXTENSION PRINCIPLES REVISITED}

Extension Principles address a fundamental problem in wavelet construction and in digital data processing. When the integer translates of a refinable function do not form a frame for their closed linear span but form only a Bessel family the construction of affine wavelet frames with desirable spatial localization cannot be carried out as in the classical multiresolution theory of Mallat and Meyer. In this case Extension Principles provide the complete answer to this problem. In addition they show that if we use the refinable function and the resulting wavelets for multiscale signal decompositions these decompositions are implementable with fast algorithms just as in the classical MRA theory. We don't want to further discuss the significance of the Extension Principles in wavelets and multiscale transforms in general. The interested reader may refer to the celebrated first paper on the topic due to Ron and Shen [61] and for nice overviews on the topic to the relevant chapter of [21] and to [26].

Let us begin with some notation. For a given refinable function $\phi$, we denote by $V_{0}$ the subspace $\overline{\operatorname{span}}\left\{T_{k} \phi\right\}_{k \in \mathbb{Z}^{d}}$. The spectrum of $\phi$, denoted by $\sigma\left(V_{0}\right)$, is defined by

$$
\sigma\left(V_{0}\right):=\left\{\xi \in \mathbb{T}^{d}: \sum_{k}|\hat{\phi}(\xi+k)|^{2}>0\right\} .
$$

The next theorem is one of the main results in [61] and is the cornerstone of the Unitary Extension Principle [61], but it also appears in [26] with different assumptions on $\phi$ where it is used to formulate the so-called Oblique Extension Principle.

Theorem 4.1. [26] Let $\phi$ be a refinable function in $L^{2}\left(\mathbb{R}^{d}\right)$ such that $\hat{\phi}$ is continuous at the origin,

$$
\lim _{|\xi| \rightarrow 0} \hat{\phi}(\xi)=1 \text {. }
$$

Assume that there exists a constant $B>0$ such that $\sum_{l \in \mathbb{Z}^{d}}|\hat{\phi}(\xi+l)|^{2} \leq B$ a.e. on $\mathbb{R}^{d}$ and that $\phi$ is refinable with respect to the expansive matrix $A$, i.e. there exists a $\mathbb{Z}^{d}$-periodic measurable function $H_{0}$, such that

$$
\hat{\phi}\left(A^{*} .\right)=H_{0} \hat{\phi}
$$


Furthermore, let $H_{i}, i=1, \ldots, m$, be $\mathbb{Z}^{d}$-periodic measurable functions and define $m$ wavelets $\psi_{i}, i=$ $1, \ldots, m$, by

$$
\hat{\psi}_{i}\left(A^{*} .\right)=H_{i} \hat{\phi} .
$$

Assume $H_{i} \in L^{\infty}\left(\mathbb{T}^{d}\right)$ for all $i=0, \ldots, m$, then the following two conditions are equivalent:

(1) The set $\left\{D_{A}^{j} T_{k} \psi_{i}: j \in \mathbb{Z}, k \in \mathbb{Z}^{d}, i=1, \ldots, m\right\}$ is a Parseval Frame for $L^{2}\left(\mathbb{R}^{d}\right)$.

(2) For all $\xi \in \sigma\left(V_{0}\right)$,

(a) $\lim _{j \rightarrow-\infty} \Theta\left(A^{* j} \xi\right)=1$.

(b) If $q \in\left(A^{*-1} \mathbb{Z}^{d}\right) / \mathbb{Z}^{d} \backslash\{0\}$ and $\xi+q \in \sigma\left(V_{0}\right)$, then

$$
\Theta\left(A^{*} \xi\right) H_{0}(\xi) \overline{H_{0}(\xi+q)}+\sum_{i=1}^{m} H_{i}(\xi) \overline{H_{i}(\xi+q)}=0 .
$$

where $\Theta$ is the so-called fundamental function, defined by

$$
\Theta(\xi)=\sum_{j=0}^{\infty} \sum_{i=1}^{m}\left|H_{i}\left(A^{* j} \xi\right)\right|^{2} \prod_{l=0}^{j-1}\left|H_{0}\left(A^{* l} \xi\right)\right|^{2} .
$$

The previous theorem characterizes all Parseval wavelet frames of $L^{2}\left(\mathbb{R}^{d}\right)$ defined by means of (11) from a refinable function satisfying (9) and (10). If we use several consecutive levels of the multiscale decomposition given by the Parseval frame $\left\{D_{A}^{j} T_{k} \psi_{i}: j \in \mathbb{Z}, k \in \mathbb{Z}^{d}, i=1, \ldots, m\right\}$ of a signal, say $f$, then it is like using a partial sum of the series

$$
\sum_{j} \sum_{k}<f, D_{A}^{j} T_{k} \psi_{i}>D_{A}^{j} T_{k} \psi_{i}=f
$$

to approximate $f$. If we are forced to take only a few levels of decomposition, then we wish to use a family augmenting the refinable function and the wavelets such as

$$
X_{\phi \psi}:=\left\{D_{A}^{j} T_{k} \psi_{i}: j \in \mathbb{N} \cup\{0\}, k \in \mathbb{Z}^{d}, i=1, \ldots, m\right\} \cup\left\{T_{k} \phi: k \in \mathbb{Z}^{d}\right\} .
$$

If this is the case, e.g. in seismic imaging [46], then it is legitimate to question whether $X_{\phi \psi}$ forms a Parseval frame of $L^{2}\left(\mathbb{R}^{d}\right)$. This problem is the focus of the present section. The answer to this problem does not follow from Theorem 4.1. However, if $X_{\phi \psi}$ is a Parseval frame of $L^{2}\left(\mathbb{R}^{d}\right)$, then it is also true that $\left\{D_{A}^{j} T_{k} \psi_{i}: j \in \mathbb{Z}, k \in \mathbb{Z}^{d}, i=1, \ldots, m\right\}$ shares the same property (see Remark 4.3 and Corollary 4.9). One of the merits of Theorem 4.7 is that it imposes minimal conditions on the filters and the refinable function $\phi$. In the spirit of Theorem 4.7, we have Theorem 4.10 which characterizes the Bessel sequence pairs $X_{\phi^{a} \psi^{a}}$ and $X_{\phi^{s} \psi^{s}}$ that are dual frames of $L^{2}\left(\mathbb{R}^{d}\right)$.

To facilitate the implementation of the decomposition of a multidimensional data set by means of a Parseval frame resulting from Theorems 4.1, 4.7, 4.4 and 4.10 we employ the modulation matrix, a concept introduced for filter design in engineering.

Definition 4.2. The modulation matrix $\mathbf{H}$ is defined by

$$
\mathbf{H}=\left(\begin{array}{cccc}
H_{0} & H_{1} & \ldots & H_{m} \\
T_{q_{1}} H_{0} & T_{q_{1}} H_{1} & \ldots & T_{q_{1}} H_{m} \\
\vdots & \vdots & \ddots & \vdots \\
T_{q_{n-1}} H_{0} & T_{q_{n-1}} H_{1} & \ldots & T_{q_{n-1}} H_{m}
\end{array}\right)
$$

where $n=\left|\operatorname{det}\left(A^{*}\right)\right|$ and $q_{l}: l=0,1,2, \ldots, n-1$ are the representatives of the quotient group $\left(A^{*-1} \mathbb{Z}^{d}\right) / \mathbb{Z}^{d}$. 
Remark 4.3. (1) A special case of Theorem 4.1 occurs when

$$
\mathbf{H H}^{*}=\left(\begin{array}{cccc}
\chi_{\sigma\left(V_{0}\right)} & 0 & \ldots & 0 \\
0 & T_{q_{1}} \chi_{\sigma\left(V_{0}\right)} & \ldots & 0 \\
\vdots & \vdots & \ddots & \vdots \\
0 & 0 & \ldots & T_{q_{n-1}} \chi_{\sigma\left(V_{0}\right)}
\end{array}\right)
$$

almost everywhere on $\mathbb{T}^{d}$, then the set $\left\{D_{A}^{j} T_{k} \psi_{i}: j \in \mathbb{Z}, k \in \mathbb{Z}^{d}, i=1, \ldots, m\right\}$ is a Parseval frame for $L^{2}\left(\mathbb{R}^{d}\right)$. In this case the fundamental function $\Theta=1$ a.e. on $\sigma\left(V_{0}\right)$. This special case is referred to as the Unitary Extension Principle in [26]. In Theorem 4.7 we show that this condition is equivalent to the set $X_{\phi \psi}$ being a Parseval frame of $L^{2}\left(\mathbb{R}^{d}\right)$. Corollary 4.9 gives that the latter property implies the conclusion of the UEP. However, our assumptions for the refinable function $\phi$ in Theorem 4.7 are more general; we neither impose a decay condition for $\hat{\phi}$ at infinity as [61] does, nor we require that the integral translates of $\phi$ form a Bessel sequence, as [26] does.

(2) If the spectrum, $\sigma\left(V_{0}\right)=\mathbb{T}^{d}$ then the condition in Eq. (13) reduces to $\mathbf{H H}^{*}=\mathbf{I}$.

(3) For a very interesting direct proof of UEP under the same more general hypotheses we use in Theorem 4.7 see [13]; see also $[9,11]$. The more general case where the fundamental function is not necessarily equal to one is referred to in [26] as the Oblique Extension Principle.

The next theorem characterizes pairs of affine families that form dual frames of $L^{2}\left(\mathbb{R}^{d}\right)$ in terms of conditions on their filters, similar to those in Theorem 4.1. The so-called Mixed Extension Principles follow directly from this theorem [26].

Theorem 4.4. [26] Let $\phi^{a}$ and $\phi^{s}$ be refinable functions in $L^{2}\left(\mathbb{R}^{d}\right)$ such that $\widehat{\phi^{a}}$ and $\widehat{\phi^{s}}$ are continuous at the origin and

$$
\lim _{|\xi| \rightarrow 0} \widehat{\phi^{a}}(\xi)=\widehat{\phi^{s}}(\xi)=1 .
$$

Let $H_{0}^{a}$ and $H_{0}^{s} \in L^{\infty}\left(\mathbb{T}^{d}\right)$, be the associated low pass filters. Furthermore, let $H_{i}^{a}, H_{i}^{s}$ for $i=1, \ldots, m$, be $\mathbb{Z}^{d}$-periodic measurable functions and define $m$ pairs of wavelets $\psi_{i}^{a}, \psi_{i}^{s} i=1, \ldots, m$, by

$$
\begin{aligned}
& \widehat{\psi_{i}^{a}}\left(A^{*} .\right)=H_{i}^{a} \hat{\phi}, \\
& \widehat{\psi_{i}^{s}}\left(A^{*} .\right)=H_{i}^{s} \hat{\phi} .
\end{aligned}
$$

Assume $H_{i}^{a}, H_{i}^{s} \in L^{\infty}\left(\mathbb{T}^{d}\right)$ for all $i=0, \ldots, m$. Then the following two conditions are equivalent,

(1) The sets $\Psi^{a}:=\left\{D_{A}^{j} T_{k} \psi_{i}^{a}: j \in \mathbb{Z}, k \in \mathbb{Z}^{d}, i=1, \ldots, m\right\}$ and $\Psi^{s}:=\left\{D_{A}^{j} T_{k} \psi_{i}^{s}: j \in \mathbb{Z}, k \in \mathbb{Z}^{d}, i=1, \ldots, m\right\}$ is a pair of dual frames for $L^{2}\left(\mathbb{R}^{d}\right)$.

(2) For all $\xi \in \sigma\left(V_{0}^{a}\right) \bigcap \sigma\left(V_{0}^{s}\right)$,

(a) $\Psi^{a}$ and $\Psi^{s}$ are Bessel families.

(b) $\lim _{j \rightarrow-\infty} \Theta_{M}\left(A^{* j} \xi\right)=1$.

(c) If $q \in\left(A^{*-1} \mathbb{Z}^{d}\right) / \mathbb{Z}^{d} \backslash\{0\}$ and $\xi+q \in \sigma\left(V_{0}^{a}\right) \bigcap \sigma\left(V_{0}^{s}\right)$, then

$$
\Theta_{M}\left(A^{*} \xi\right) H_{0}^{s}(\xi) \overline{H_{0}^{a}(\xi+q)}+\sum_{i=1}^{m} H_{i}^{s}(\xi) \overline{H_{i}^{a}(\xi+q)}=0 .
$$

where $\Theta_{M}$ is the so-called Mixed Fundamental function, defined by

$$
\Theta_{M}(\xi)=\sum_{j=0}^{\infty} \sum_{i=1}^{m} H_{i}^{s}\left(A^{* j} \xi\right) \overline{H_{i}^{a}\left(A^{* j} \xi\right)} \prod_{l=0}^{j-1} H_{0}^{s}\left(A^{* l} \xi\right) \overline{H_{0}^{a}\left(A^{* l} \xi\right)} .
$$


Remark 4.5. Let $\mathbf{H}_{a}$ and $\mathbf{H}_{s}$ denote the modulation matrices corresponding to the filters $H_{i}^{a}$ and $H_{i}^{s}$ respectively. By Theorem 4.4 we conclude that if

$$
\mathbf{H}_{s} \mathbf{H}_{a}^{*}=\left(\begin{array}{cccc}
\chi_{\sigma\left(V_{0}^{a}\right) \cap} \cap\left(V_{0}^{s}\right) & 0 & \cdots & 0 \\
0 & T_{q_{1}} \chi_{\sigma\left(V_{0}^{a}\right) \cap \sigma\left(V_{0}^{s}\right)} & \cdots & 0 \\
\vdots & \vdots & \ddots & \vdots \\
0 & 0 & \ldots & T_{q_{n-1}} \chi_{\sigma\left(V_{0}^{a}\right) \cap \sigma\left(V_{0}^{s}\right)}
\end{array}\right)
$$

holds almost everywhere on $\mathbb{T}^{d}$ then $\Psi^{a}$ and $\Psi^{s}$ is a pair of dual frames for $L^{2}\left(\mathbb{R}^{d}\right)$. This special case of Theorem 4.4 is referred to as the Mixed Extension Principle in [26]. Notice that in this case, the Mixed Fundamental function $\Theta_{M}=1$ a.e. on $\sigma\left(V_{0}^{a}\right) \bigcap \sigma\left(V_{0}^{s}\right)$. The more general case where $\Theta_{M}$ may take values other than one is referred to as the Mixed Oblique Extension Principle in [26].

The purpose of the next theorem is to characterize when the set $X_{\phi \psi}$ is a Parseval frame for $L^{2}\left(\mathbb{R}^{d}\right)$ under the most general hypotheses on the refinable function $\phi$ and on the filters $H_{i}$. Before we state and prove the theorem, let us prove the following lemma required in the proof of the theorem and also for the examples described in Section 5.

Lemma 4.6. Let $G$ be a finite group with the group operation denoted by $\circ$ and let $\chi$ be a character on $G$. If $\chi$ is not the identity character on $G$, i.e. there exists an $h$ in $G$ such that $\chi(h) \neq 1$, then $\sum_{g \in G} \chi(g)=0$.

Proof. Let $h$ in $G$ satisfying $\chi(h) \neq 1$. Set $Y:=\sum_{g \in G} \chi(g)$ and observe

$$
\chi(h) \sum_{g \in G} \chi(g)=\sum_{g \in G} \chi(h \circ g)=Y .
$$

Hence, we have $Y(\chi(h)-1)=0$, which gives us the required result since $\chi(h) \neq 1$.

Theorem 4.7. Let $\phi \in L^{2}\left(\mathbb{R}^{d}\right)$ be a refinable function such that $\hat{\phi}$ is continuous at the origin satisfying Eqs. (9) and (10). Moreover assume $H_{i} \in L^{\infty}\left(\mathbb{T}^{d}\right)$ for all $i=1, \ldots, m$ are $\mathbb{Z}^{d}$-periodic functions and $\psi_{i} \in L^{2}\left(\mathbb{R}^{d}\right)$ are given by $E q$. (11). Then $X_{\phi \psi}$ is a Parseval frame for $L^{2}\left(\mathbb{R}^{d}\right)$ if and only if for all $q \in\left(A^{*-1} \mathbb{Z}^{d}\right) / \mathbb{Z}^{d}$ and for a.e. $\xi, \xi+q \in \sigma\left(V_{0}\right)$,

$$
\sum_{i=0}^{m} H_{i}(\xi) \overline{H_{i}(\xi+q)}=\delta_{q, 0} .
$$

Proof. The proof splits in two parts. In the first part, we establish that $X_{\phi \psi}$ is a Parseval frame if and only if the following condition holds,

$$
\sum_{j=0}^{j_{0}(l)} \sum_{i=1}^{m} \widehat{\psi}_{i}\left(A^{*-j} \xi\right) \overline{\widehat{\psi}_{i}\left(A^{*-j}(\xi+l)\right)}+\widehat{\phi}(\xi) \overline{\widehat{\phi}(\xi+l)}=\delta_{l, 0} .
$$

for a.e. $\xi \in \mathbb{R}^{d}$ and $l \in \mathbb{Z}^{d}$ (The function $j_{0}$ is defined in (21) below). In the second part, we show that this condition is equivalent to (18). First, assume that $X_{\phi \psi}$ is a Parseval frame for $L^{2}\left(\mathbb{R}^{d}\right)$ and obtain the above condition.

We begin by defining a unitary operator $U: L^{2}\left(\mathbb{R}^{d}\right) \rightarrow L^{2}\left(\mathbb{T}^{d}, l^{2}\left(\mathbb{Z}^{d}\right)\right)$ via

$$
U(f)=\left\{\widehat{f}(.+k): k \in \mathbb{Z}^{d}\right\} .
$$

Next we define the set $J$ by

$$
J:=\left\{(j, r, i): j \in \mathbb{N} \cup\{0\}, r \in \mathbb{Z}^{d} /\left(A^{* j} \mathbb{Z}^{d}\right), i=1, \ldots, m\right\} \cup\{(0,0,0)\},
$$

and a map $S: L^{2}\left(\mathbb{T}^{d}, l^{2}(J)\right) \rightarrow L^{2}\left(\mathbb{T}^{d}, l^{2}\left(\mathbb{Z}^{d}\right)\right)$, via

$$
S\left(e_{l}, \delta_{(j, r, i)}\right)=U\left(T_{l} D^{j} T_{r} \psi_{i}\right) \quad \text { for all } l \in \mathbb{Z}^{d},(j, r, i) \in J
$$


where we adopt the convention $\psi_{0}=\phi$. Note that,

$$
\left(S e_{l} \delta_{(j, r, i)}\right)(\xi)=\left\{e_{l}(\xi)|\operatorname{det}(A)|^{-j / 2} e^{-2 \pi i\left\langle r, A^{*-j}(\xi+k)\right\rangle} \widehat{\psi}_{i}\left(A^{*-j}(\xi+k)\right)\right\}_{k \in \mathbb{Z}^{d}} \text { for a.e. } \xi \in \mathbb{T}^{d} .
$$

Since $X_{\phi \psi}$ is a Parseval frame, $S$ can be extended to a bounded linear transformation on $L^{2}\left(\mathbb{T}^{d}, l^{2}(J)\right)$. Now $S$ can be represented by a $\mathbb{Z}^{d} \times J$ matrix so that the $(k,(j, r, i))$-th entry of the matrix is an operator on $L^{2}\left(\mathbb{T}^{d}\right)$, denoted by $S_{k,(j, r, i)}$. This operator acts on the modulations as follows:

$$
\left(S_{k,(j, r, i)} e_{l}\right)(\xi)=e_{l}(\xi)|\operatorname{det}(A)|^{-j / 2} e^{-2 \pi i\left\langle r,\left(A^{*-j}(\xi+k)\right)\right\rangle} \widehat{\psi}_{i}\left(A^{*-j}(\xi+k)\right) .
$$

Thus we see that each $S_{k,(j, r, i)}$ commutes with all the modulation operators $M_{l}$ defined on $L^{2}\left(\mathbb{T}^{d}\right)$ by $M_{l} \omega(\xi):=e_{l}(\xi) \omega(\xi)$. Hence, each $S_{k,(j, r, i)}$ is a multiplicative operator (e.g. [25, Corollary 2.12.7]) in the following sense:

$$
\left(S_{k,(j, r, i)} \omega\right)(\xi)=\omega(\xi)|\operatorname{det}(A)|^{-j / 2} e^{-2 \pi i\left\langle r,\left(A^{*-j}(\xi+k)\right)\right\rangle} \widehat{\psi}_{i}\left(A^{*-j}(\xi+k)\right),
$$

for all $\omega \in L^{2}\left(\mathbb{T}^{d}\right)$. We denote the symbol for this operator again by $S_{k,(j, r, i)}(\cdot)$, so that

$$
S_{k,(j, r, i)}(\xi)=|\operatorname{det}(A)|^{-j / 2} e^{-2 \pi i\left\langle r,\left(A^{*-j}(\xi+k)\right)\right\rangle} \widehat{\psi}_{i}\left(A^{*-j}(\xi+k)\right) .
$$

Note that $S_{k,(j, r, i)}(\cdot)$ is an essentially bounded $\mathbb{Z}^{d}$-periodic function. Let $S($.$) denote the \mathbb{Z}^{d} \times J$ matrix of the symbols $S_{k,(j, r, i)}($.$) , then$

$$
(S \omega)(\xi)=S(\xi) \omega(\xi) \quad \text { for all } \omega \in L^{2}\left(\mathbb{T}^{d}, l^{2}(J)\right) .
$$

Also $\|S()$.$\| is essentially bounded [25, Theorem 7.52.8] Now, the adjoint of S$ can be represented by the $J \times \mathbb{Z}^{d}$ matrix of operators on $L^{2}\left(\mathbb{T}^{d}\right)$ such that the $((j, r, i), k)$-th entry is $S_{k,(j, r, i)}^{*}$. Since $S_{k,(j, r, i)}^{*}$ is the adjoint of a multiplicative operator, it is itself a multiplicative operator with symbol $\overline{S_{k,(j, r, i)}(.)}$. Therefore,

$$
\left(S^{*} \omega\right)(\xi)=S(\xi)^{*} \omega(\xi) \quad \text { for all } \omega \in L^{2}\left(\mathbb{T}^{d}, l^{2}\left(\mathbb{Z}^{d}\right)\right)
$$

Since $X_{\phi \psi}$ is a Parseval frame, we have, $S S^{*}=I_{L^{2}\left(\mathbb{T}^{d}, l^{2}\left(\mathbb{Z}^{d}\right)\right)}$, i.e.

$$
S(\xi) S(\xi)^{*}=I_{l^{2}\left(\mathbb{Z}^{d}\right)} \quad \text { for a.e. } \xi \in \mathbb{T}^{d} .
$$

The $(k, l)$-th entry of $S(\xi) S(\xi)^{*}$ is given by

$$
\begin{aligned}
\left(S(\xi) S(\xi)^{*}\right)_{(k, l)}= & \sum_{j=0}^{\infty} \sum_{i=1}^{m}|\operatorname{det}(A)|^{-j} \sum_{r \in \mathbb{Z}^{d} /\left(A^{* j} \mathbb{Z}^{d}\right)} e^{2 \pi i\left\langle r, A^{*-j}(k-l)\right\rangle} \widehat{\psi}_{i}\left(A^{*-j}(\xi+k)\right) \overline{\widehat{\psi}_{i}\left(A^{*-j}(\xi+l)\right)} \\
& +\widehat{\phi}(\xi+k) \overline{\widehat{\phi}(\xi+l) .} .
\end{aligned}
$$

Now, define $j_{0}: \mathbb{Z}^{d} \backslash\{0\} \rightarrow \mathbb{Z}$ via

$$
j_{0}(l)=\sup \left\{j: A^{*-j}(l) \in \mathbb{Z}^{d}\right\} .
$$

Obviously, if $l \neq 0$, then $j_{0}(l)$ is finite while $j_{0}(0)$ can be set equal to $+\infty$. If $j>j_{0}(k-l)$ then $A^{*-j}(k-l) \notin \mathbb{Z}^{d}$ and therefore $e_{A^{*-j}(k-l)}$ is not the identity character on $\mathbb{Z}^{d} /\left(A^{* j} \mathbb{Z}^{d}\right)$. On the other hand, if $j \leq j_{0}(k-l)$ then $A^{*-j}(k-l) \in \mathbb{Z}^{d}$ and $e_{A^{*-j}(k-l)}$ is the identity character on $\mathbb{Z}^{d} /\left(A^{* j} \mathbb{Z}^{d}\right)$. Thus using Lemma 4.6, we conclude

$$
|\operatorname{det}(A)|^{-j} \sum_{r \in \mathbb{Z}^{d} /\left(A^{* j} \mathbb{Z}^{d}\right)} e^{2 \pi i\left\langle r, A^{*-j}(k-l)\right\rangle}=\left\{\begin{array}{ll}
0 & \text { for } j>j_{0}(k-l) \\
1 & \text { for } j \leq j_{0}(k-l)
\end{array} .\right.
$$

Hence, for $k \neq l$, Eq. (20) reduces to,

$$
\left(S(\xi) S(\xi)^{*}\right)_{(k, l)}=\sum_{j=0}^{j_{0}(k-l)} \sum_{i=1}^{m} \widehat{\psi}_{i}\left(A^{*-j}(\xi+k)\right) \overline{\widehat{\psi}_{i}\left(A^{*-j}(\xi+l)\right)}+\widehat{\phi}(\xi+k) \overline{\widehat{\phi}(\xi+l)} .
$$


Thus using Eq. (19), we conclude that the following condition holds for a.e. $\xi \in \mathbb{T}^{d}$ and $k, l \in \mathbb{Z}^{d}$ :

$$
\sum_{j=0}^{j_{0}(k-l)} \sum_{i=1}^{m} \widehat{\psi}_{i}\left(A^{*-j}(\xi+k)\right) \overline{\widehat{\psi}_{i}\left(A^{*-j}(\xi+l)\right)}+\widehat{\phi}(\xi+k) \overline{\widehat{\phi}(\xi+l)}=\delta_{k, l} .
$$

By changing variables the previous equation becomes equivalent to:

$$
\sum_{j=0}^{j_{0}(l)} \sum_{i=1}^{m} \widehat{\psi}_{i}\left(A^{*-j} \xi\right) \overline{\widehat{\psi}_{i}\left(A^{*-j}(\xi+l)\right)}+\widehat{\phi}(\xi) \overline{\widehat{\phi}(\xi+l)}=\delta_{0, l} .
$$

for a.e. $\xi \in \mathbb{R}^{d}$ and $l \in \mathbb{Z}^{d}$.

Conversely, we must show that if (23) holds for a.e. $\xi \in \mathbb{R}^{d}$ and $l \in \mathbb{Z}^{d}$, then $X_{\phi \psi}$ is a Parseval frame. To this end, we densely define the operators $\mathcal{A}(\xi): l^{2}\left(\mathbb{Z}^{d}\right) \rightarrow l^{2}(J)$ via

$$
\mathcal{A}(\xi) \delta_{k}=\left\{|\operatorname{det}(A)|^{-j / 2} e^{2 \pi i\left\langle r, A^{*-j}(\xi+k)\right\rangle}{\overline{\widehat{\psi}_{i}}\left(A^{*-j}(\xi+k)\right)}_{\}_{(j, r, i) \in J}} .\right.
$$

Using (22) which is equivalent to (23), we conclude that $\mathcal{A}(\xi)$ extends to an isometry on $l^{2}\left(\mathbb{Z}^{d}\right)$, for a.e. $\xi \in \mathbb{T}^{d}$. We now define the operator $\mathcal{A}: L^{2}\left(\mathbb{T}^{d}, l^{2}\left(\mathbb{Z}^{d}\right)\right) \rightarrow L^{2}\left(\mathbb{T}^{d}, l^{2}(J)\right)$, via

$$
(\mathcal{A} \omega)(\xi)=\mathcal{A}(\xi) \omega(\xi) \text {. }
$$

Since $\mathcal{A}(\xi)$ is an isometry for a.e. $\xi \in \mathbb{T}^{d}$, it is easy to verify that $\mathcal{A}$ is an isometry. But $\mathcal{A}$ coincides with $S^{*}$, which is the analysis operator for the family $X_{\phi \psi}$. Therefore $X_{\phi \psi}$ is a Parseval frame, finishing the first part of the proof.

Now, we proceed to the second part of the proof. In this part we show that (23) holds if and only if (18) holds. We will first assume (18) holds and prove that (23) holds for all $l \neq 0$. Note, that $A^{*-j} l \in \mathbb{Z}^{d}$ for all $j=0,1, \ldots, j_{0}(l)$. Thus, for all these $j$ we have $\sum_{i=0}^{m} H_{i}\left(A^{*-\left(j_{0}(l)+1\right)} \xi\right) \overline{H_{i}\left(A^{*-\left(j_{0}(l)+1\right)}(\xi+l)\right)}=1$ due to Eq. (18). Using the last equation and the two-scale relations ((10) and (11)) we obtain,

$$
\begin{aligned}
& \sum_{j=0}^{j_{0}(l)} \sum_{i=1}^{m} \widehat{\psi}_{i}\left(A^{*-j} \xi\right) \overline{\widehat{\psi}_{i}\left(A^{*-j}(\xi+l)\right)}+\widehat{\phi}(\xi) \overline{\widehat{\phi}(\xi+l)}=\sum_{j=1}^{j_{0}(l)} \sum_{i=1}^{m} \widehat{\psi}_{i}\left(A^{*-j} \xi\right) \overline{\widehat{\psi}_{i}\left(A^{*-j}(\xi+l)\right)}+ \\
& +\widehat{\phi}\left(A^{*-1} \xi\right) \overline{\widehat{\phi}\left(A^{*-1}(\xi+l)\right)}\left(\sum_{i=0}^{m} H_{i}\left(A^{*-1} \xi\right) \overline{H_{i}\left(A^{*-1}(\xi+l)\right)}\right) \\
& =\sum_{j=1}^{j_{0}(l)} \sum_{i=1}^{m} \widehat{\psi}_{i}\left(A^{*-j} \xi\right) \overline{\widehat{\psi}_{i}\left(A^{*-j}(\xi+l)\right)}+\widehat{\phi}\left(A^{*-1} \xi\right) \overline{\widehat{\phi}\left(A^{*-1}(\xi+l)\right)} \\
& =\sum_{i=1}^{m} \widehat{\psi}_{i}\left(A^{*-j_{0}(l)} \xi\right) \overline{\widehat{\psi}_{i}\left(A^{*-j_{0}(l)}(\xi+l)\right)} \\
& +\widehat{\phi}\left(A^{*-j_{0}(l)} \xi\right) \overline{\widehat{\phi}\left(A^{*-j_{0}(l)}(\xi+l)\right)}\left(\sum_{i=0}^{m} H_{i}\left(A^{*-j_{0}(l)} \xi\right) \overline{H_{i}\left(A^{*-j_{0}(l)}(\xi+l)\right)}\right) \\
& =\sum_{i=1}^{m} \widehat{\psi}_{i}\left(A^{*-j_{0}(l)} \xi\right) \overline{\widehat{\psi}_{i}\left(A^{*-j_{0}(l)}(\xi+l)\right)}+\widehat{\phi}\left(A^{*-j_{0}(l)} \xi\right) \overline{\widehat{\phi}\left(A^{*-j_{0}(l)}(\xi+l)\right)} \\
& =\widehat{\phi}\left(A^{*-\left(j_{0}(l)+1\right)} \xi\right) \overline{\widehat{\phi}\left(A^{*-\left(j_{0}(l)+1\right)}(\xi+l)\right)}\left(\sum_{i=0}^{m} H_{i}\left(A^{*-\left(j_{0}(l)+1\right)} \xi\right) \overline{H_{i}\left(A^{*-\left(j_{0}(l)+1\right)}(\xi+l)\right)}\right)=0 .
\end{aligned}
$$


Next we show that (23) holds for $l=0$, using the hypothesis (18). For a fixed $N$ we have

$$
\begin{aligned}
\sum_{j=0}^{N} \sum_{i=1}^{m}\left|\widehat{\psi}_{i}\left(A^{*-j} \xi\right)\right|^{2}+|\widehat{\phi}(\xi)|^{2} & =\sum_{j=1}^{N} \sum_{i=1}^{m}\left|\widehat{\psi}_{i}\left(A^{*-j} \xi\right)\right|^{2}+\left|\widehat{\phi}\left(A^{*-1} \xi\right)\right|^{2}\left(\sum_{i=0}^{m}\left|H_{i}\left(A^{*-1} \xi\right)\right|^{2}\right) \\
& =\sum_{j=1}^{N} \sum_{i=1}^{m}\left|\widehat{\psi}_{i}\left(A^{*-j} \xi\right)\right|^{2}+\left|\widehat{\phi}\left(A^{*-1} \xi\right)\right|^{2} \\
& =\sum_{j=2}^{N} \sum_{i=1}^{m}\left|\widehat{\psi}_{i}\left(A^{*-j} \xi\right)\right|^{2}+\left|\widehat{\phi}\left(A^{*-2} \xi\right)\right|^{2}\left(\sum_{i=0}^{m}\left|H_{i}\left(A^{*-2} \xi\right)\right|^{2}\right) \\
& =\sum_{j=2}^{N} \sum_{i=1}^{m}\left|\widehat{\psi}_{i}\left(A^{*-j} \xi\right)\right|^{2}+\left|\widehat{\phi}\left(A^{*-2} \xi\right)\right|^{2} \\
& \vdots \\
& =\sum_{i=1}^{m}\left|\widehat{\psi}_{i}\left(A^{*-N} \xi\right)\right|^{2}+\left|\widehat{\phi}\left(A^{*-N} \xi\right)\right|^{2}\left(\sum_{i=0}^{m}\left|H_{i}\left(A^{*-N} \xi\right)\right|^{2}\right) \\
& =\sum_{i=1}^{m}\left|\widehat{\psi}_{i}\left(A^{*-N} \xi\right)\right|^{2}+\left|\widehat{\phi}\left(A^{*-N} \xi\right)\right|^{2} \\
& =\left|\widehat{\phi}\left(A^{*-N-1} \xi\right)\right|^{2}\left(\sum_{i=0}^{m}\left|H_{i}\left(A^{*-N-1} \xi\right)\right|^{2}\right)=\left|\widehat{\phi}\left(A^{*-N-1} \xi\right)\right|^{2}
\end{aligned}
$$

Now the assumption (9), together with the continuity of $\widehat{\phi}$ at 0 imply that for every $\epsilon>0$, there exists a $N_{0}$ such that $\left.|| \widehat{\phi}\left(A^{*-N} \xi\right)\right|^{2}-1 \mid<\epsilon$ for all $N>N_{0}$. This in turn implies that

$$
\left.\left|\sum_{j=0}^{N} \sum_{i=1}^{m}\right| \widehat{\psi}_{i}\left(A^{*-j} \xi\right)\right|^{2}+|\widehat{\phi}(\xi)|^{2}-1 \mid<\epsilon
$$

for all $N>N_{0}+1$. This proves that (23) holds for $l=0$. Thus, we have shown that (18) implies (23).

To establish the converse implication, first notice that the calculations we carried out to prove that Eq. (18) implies Eq. (23) for $l \neq 0$ are still valid for almost every $\xi$ and all $l \in \mathbb{Z}^{d}$. Now pick $q \in\left(A^{*-1}\right) \mathbb{Z}^{d} / \mathbb{Z}^{d}$. We have $A^{*} q \in \mathbb{Z}^{d}$. If $p$ is an arbitrary integer grid point, i.e. $p \in \mathbb{Z}^{d}$ set $l:=A^{*}(q+p)$. Obviously $l \in \mathbb{Z}^{d}$, since $A$ is expansive and thus $A^{*}$ leaves the lattice $\mathbb{Z}^{d}$ invariant. Observe $j_{0}(l)=0$, because $p+q$ does not belong to the integer grid. Next, assume that (18) holds for $q=0$. Since, (23) is valid for all $l \neq 0$ we deduce

$$
\begin{aligned}
0 & =\sum_{i=1}^{m} \widehat{\psi}_{i}(\xi) \overline{\widehat{\psi}_{i}(\xi+l)}+\widehat{\phi}(\xi) \overline{\widehat{\phi}(\xi+l)} \\
& =\widehat{\phi}\left(A^{*-1} \xi\right) \overline{\widehat{\phi}\left(A^{*-1}(\xi+l)\right)}\left(\sum_{i=0}^{m} H_{i}\left(A^{*-1} \xi\right) \overline{H_{i}\left(A^{*-1}(\xi+l)\right)}\right) \\
& =\widehat{\phi}\left(A^{*-1} \xi\right) \overline{\widehat{\phi}\left(A^{*-1} \xi+q+p\right)}\left(\sum_{i=0}^{m} H_{i}\left(A^{*-1} \xi\right) \overline{H_{i}\left(A^{*-1} \xi+q+p\right)}\right) \text { a.e. in } \mathbb{R}^{d}
\end{aligned}
$$

Take $\xi$ and $\xi+q$ in $\sigma\left(V_{0}\right)$ such that $A^{*} \xi$ belongs to the set of points in $\mathbb{R}^{d}$ for which Eq. (23) holds. The fact that Eq. (23) is equivalent to Eq. (22) implies that, if Eq. (23) holds for a $\xi \in \mathbb{R}^{d}$ then, Eq. (23) holds for 
all integer translates of this point. Apply the previous equations for $A^{*}(\xi+s)$ instead of $\xi$, where $s \in \mathbb{Z}^{d}$. Then

$$
0=\widehat{\phi}(\xi+s) \overline{\widehat{\phi}(\xi+q+p)}\left(\sum_{i=0}^{m} H_{i}(\xi) \overline{H_{i}(\xi+q)}\right)
$$

due to the $\mathbb{Z}^{d}$-periodicity of $H_{i}$. Since $p, s$ are arbitrary integers and $\xi, \xi+q \in \sigma\left(V_{0}\right)$ there exist some $s_{0}$ and $p_{0}$ for which

$$
\widehat{\phi}\left(\xi+s_{0}\right) \widehat{\phi}\left(\xi+q+p_{0}\right) \neq 0 \text {. }
$$

Thus,

$$
\sum_{i=0}^{m} H_{i}(\xi) \overline{H_{i}(\xi+q)}=0 .
$$

In order to complete the proof of the theorem we need to establish that (18) holds for $q=0$. To verify this, set $l=0$ in (23):

$$
\sum_{j=0}^{\infty} \sum_{i=1}^{m}\left|\widehat{\psi}_{i}\left(A^{*-j} \xi\right)\right|^{2}+|\widehat{\phi}(\xi)|^{2}=1, \quad \xi \in \mathbb{R}^{d} \text { a.e. . }
$$

Observe that this implies

$$
\sum_{j=0}^{\infty} \sum_{i=1}^{m}\left|\widehat{\psi}_{i}\left(A^{*-j+1} \xi\right)\right|^{2}+\left|\widehat{\phi}\left(A^{*} \xi\right)\right|^{2}=1, \quad \xi \in \mathbb{R}^{d} \text { a.e. . }
$$

Using the two scale relations, (10) and (11), we infer

$$
\sum_{j=0}^{\infty} \sum_{i=1}^{m}\left|\widehat{\psi}_{i}\left(A^{*-j+1} \xi\right)\right|^{2}+\left|\widehat{\phi}\left(A^{*} \xi\right)\right|^{2}=\sum_{j=0}^{\infty} \sum_{i=1}^{m}\left|\widehat{\psi}_{i}\left(A^{*-j} \xi\right)\right|^{2}+|\widehat{\phi}(\xi)|^{2}\left(\sum_{i=0}^{m}\left|H_{i}(\xi)\right|^{2}\right) .
$$

Hence,

$$
\sum_{j=0}^{\infty} \sum_{i=1}^{m}\left|\widehat{\psi}_{i}\left(A^{*-j} \xi\right)\right|^{2}+|\widehat{\phi}(\xi)|^{2}=\sum_{j=0}^{\infty} \sum_{i=1}^{m}\left|\widehat{\psi}_{i}\left(A^{*-j} \xi\right)\right|^{2}+|\widehat{\phi}(\xi)|^{2}\left(\sum_{i=0}^{m}\left|H_{i}(\xi)\right|^{2}\right),
$$

Therefore, $\sum_{i=0}^{m}\left|H_{i}(\xi)\right|^{2}=1$ for a.e. $\xi \in \sigma\left(V_{0}\right)$.

Remark 4.8. The characterization of affine frames in [61] utilizes

$$
\Psi\left[\omega, \omega^{\prime}\right]:=\sum_{\psi \in \Psi} \sum_{j=\kappa\left(\omega-\omega^{\prime}\right)}^{\infty} \widehat{\psi}\left(2^{j} \omega\right) \overline{\hat{\psi}\left(2^{j} \omega^{\prime}\right)}, \quad \omega, \omega^{\prime} \in \mathbb{R}
$$

where $\kappa$ is defined by $\kappa(\omega)=\inf \left\{j \in \mathbb{Z}: 2^{j} \omega \in 2 \pi \mathbb{Z}\right\}$. A similar convention is used by Bownik [17]. This is reminiscent of the term of the sum in the left-hand side of Eq. (23) involving the wavelets $\psi_{i}$. However, since we use positive powers of $D_{A}$ in the definition of $X_{\phi \psi}$, we arrive at negative powers of $A^{*}$.

An immediate consequence of the previous theorem is a generalization of the UEP.

Corollary 4.9. [13] Under the hypotheses of Theorem 4.7 the following is true: If Condition18 holds for all $q \in\left(A^{*-1} \mathbb{Z}^{d}\right) / \mathbb{Z}^{d}$ and for a.e. $\xi, \xi+q \in \sigma\left(V_{0}\right)$, then the set $\left\{D_{A}^{j} T_{k} \psi_{i}: j \in \mathbb{Z}, k \in \mathbb{Z}^{d}, i=1, \ldots, m\right\}$ is a Parseval frame for $L^{2}\left(\mathbb{R}^{d}\right)$. 
Proof. Obviously we have that $X_{\phi \psi}$ is a Parseval frame of $L^{2}\left(\mathbb{R}^{d}\right)$, thus for every $j_{o} \in \mathbb{Z}$ the set $\left\{D_{A}^{j} T_{k} \psi_{i}: j \geq j_{0}, k \in \mathbb{Z}^{d}, i=1, \ldots, m\right\} \cup\left\{D_{A}^{j_{0}} T_{k} \phi: k \in \mathbb{Z}^{d}\right\}$ is a Parseval frame of $L^{2}\left(\mathbb{R}^{d}\right)$ as well. Now, take $f \in L^{2}\left(\mathbb{R}^{d}\right)$. Then as $j_{0} \rightarrow-\infty$ observe that

$$
\lim _{j_{0} \rightarrow-\infty} \sum_{k \in \mathbb{Z}^{d}}\left|<f, D_{A}^{j_{0}} T_{k} \phi>\right|^{2}=0 .
$$

The proof of this fact is not hard (see [13, Lemma 7.7]), so we omit it.

Theorem 4.7 shows that $X_{\phi \psi}$ is a Parseval frame if and only if the conditions in Theorem 4.1 hold with the fundamental function equal to 1 a.e. on $\sigma\left(V_{0}\right)$. In the same spirit, the next theorem characterizes the Bessel families, $X_{\phi \psi}^{a}$ and $X_{\phi \psi}^{s}$, that form a pair of dual frames based on conditions on the corresponding filters. We infer that $X_{\phi \psi}^{a}$ and $X_{\phi \psi}^{s}$ are a pair of dual frames for $L^{2}\left(\mathbb{R}^{d}\right)$ if and only if the conditions in Theorem 4.4 hold with the mixed fundamental function equal to 1 a.e. on $\sigma\left(V_{0}^{a}\right) \cap \sigma\left(V_{0}^{s}\right)$. The proof of Theorem 4.10 follows from arguments similar to those in the proof of Theorem 4.7. We only give the statement here. For a complete proof of this result the reader is referred to [46].

Theorem 4.10. Let $\phi^{a}$ and $\phi^{s}$ be refinable functions in $L^{2}\left(\mathbb{R}^{d}\right)$ such that $\hat{\phi}^{a}$ and $\hat{\phi}^{s}$ are continuous at the origin and

$$
\lim _{|\xi| \rightarrow 0} \widehat{\phi^{a}}(\xi)=\widehat{\phi^{s}}(\xi)=1 .
$$

Let $H_{0}^{a}$ and $H_{0}^{s} \in L^{\infty}\left(\mathbb{T}^{d}\right)$, be the associated low pass filters. Furthermore, let $H_{i}^{a}, H_{i}^{s} \in L^{\infty}\left(\mathbb{T}^{d}\right)$ for $i=1, \ldots, m$, be $\mathbb{Z}^{d}$-periodic functions and define $m$ pairs of wavelets $\psi_{i}^{a}, \psi_{i}^{s} i=1, \ldots, m$, by

$$
\begin{aligned}
& \widehat{\psi_{i}^{a}}\left(A^{*} .\right)=H_{i}^{a} \widehat{\phi^{a}} . \\
& \widehat{\psi_{i}^{s}}\left(A^{*} .\right)=H_{i}^{s} \widehat{\phi^{s}} .
\end{aligned}
$$

Then $X_{\phi \psi}^{a}$ and $X_{\phi \psi}^{s}$ form a pair of dual frames for $L^{2}\left(\mathbb{R}^{d}\right)$ if and only if

(1) $X_{\phi \psi}^{a}$ and $X_{\phi \psi}^{s}$ are Bessel families,

(2) For all $q \in\left(A^{*-1} \mathbb{Z}^{d}\right) / \mathbb{Z}^{d}$ and for a.e. $\xi, \xi+q \in \sigma\left(V_{0}^{a}\right) \cap \sigma\left(V_{0}^{s}\right)$,

$$
\sum_{i=0}^{m} H_{i}^{s}(\xi) \overline{H_{i}^{a}(\xi+q)}=\delta_{q, 0} .
$$

\section{EXAMPLES OF ISOTROPIC WAVELET FrAMES}

In this section we describe two examples of Isotropic wavelet frames. With the help of Theorems 4.1, 4.7, 4.4 and 4.10, the problem of designing wavelet frames is transformed into a problem of designing the corresponding filters. We illustrate this in Examples 5.1 and 5.2. Example 5.1 is an example of a Parseval frame while Example 5.2 is an example of a pair of dual frames. Recall that we define the dilation operator with respect to a radially expansive matrix $A=a \mathcal{O}$, where $\mathcal{O}$ is a rotation. The constant $a>0$ will be used in the examples below.

Example 5.1. As pointed out at the beginning of this section, we obtain the wavelet frame, by designing the corresponding filters and then invoking the appropriate extension principle. We begin with the low pass filter $H_{0}$, a smooth $\mathbb{Z}^{d}$-periodic function such that

- $H_{0}=1$ inside the ball of radius $b_{1}$

- $H_{0}=0$ on $\mathbb{T}^{d} \backslash B\left(0, b_{0}\right)$

- $\left.H_{0}\right|_{B\left(0, b_{0}\right)}$ is radial, 
where $\frac{1}{2 a^{2}}<b_{1}<b_{0}<\frac{1}{2 a}$.

If $\phi$ by $\hat{\phi}=H_{0}\left(A^{*-1}\right.$.) $\chi_{\mathbb{T}^{d}}$, then $\phi$ satisfies conditions (9) and (10) of Theorem 4.1.

Now, let $h$ be a $\mathbb{Z}^{d}$-periodic function defined by,

$$
h(\xi)=\sqrt{\frac{1-H_{0}^{2}(\xi)}{|\operatorname{det}(A)|}} .
$$

Using $h$ we define the high pass filters as follows,

$$
H_{i}(\xi)=e_{q_{i-1}}(\xi) h(\xi), \quad i=1, \ldots,|\operatorname{det}(A)|,
$$

where $\left\{q_{l}: l=0,1,2, \ldots,|\operatorname{det}(A)|-1\right\}$ are the representatives of the quotient group $\mathbb{Z}^{d} /\left(A^{*} \mathbb{Z}^{d}\right)$.

We claim $\mathbf{H H}^{*}=\mathbf{I}$, where $\mathbf{H}$ is defined by (12). Hence, by Theorem 4.1, the set

$$
\left\{D^{j} T_{k} \psi_{i}: j \in \mathbb{Z}, k \in \mathbb{Z}^{d}, i=1, \ldots,|\operatorname{det}(A)|\right\}
$$

forms a Parseval Frame for $L^{2}\left(\mathbb{R}^{d}\right)$. Also, by Theorem 4.7, the set $X_{\phi \psi}$ is a Parseval frame for $L^{2}\left(\mathbb{R}^{d}\right)$.

To prove our claim, we use (27) and (28) to obtain

$$
\left(\mathbf{H H}^{*}\right)_{1,1}(\xi)=\sum_{i=0}^{|\operatorname{det}(A)|}\left|H_{i}(\xi)\right|^{2}=H_{0}^{2}(\xi)+|\operatorname{det}(A)| \frac{1-H_{0}^{2}(\xi)}{|\operatorname{det}(A)|}=1 \text { for all } \xi \in \mathbb{T}^{d} .
$$

and

$$
\left(\mathbf{H H}^{*}\right)_{i+1, i+1}(\xi)=\left(\mathbf{H H}^{*}\right)_{1,1}\left(\xi+A^{*-1} q_{i-1}\right)=1 \text { for all } \xi \in \mathbb{T}^{d}, i=1, \ldots,|\operatorname{det}(A)| \text {. }
$$

Next we want to show that the off-diagonal entries of $\mathbf{H H}^{*}$ are zero. To do this we observe that the supports of $H_{0}\left(.+A^{*-1} q_{i-1}\right)$ and $\overline{H_{0}\left(.+A^{*-1} q_{j-1}\right)}$ are disjoint by definition of $H_{0}$. Thus,

$$
H_{0}\left(\xi+A^{*-1} q_{i-1}\right) \overline{H_{0}\left(\xi+A^{*-1} q_{j-1}\right)}=0 \quad \text { for all } \xi \in \mathbb{T}^{d}, i \neq j .
$$

Hence,

$$
\begin{aligned}
\left(\mathbf{H H}^{*}\right)_{j, k}(\xi) & =\sum_{i=0}^{|\operatorname{det}(A)|} H_{i}\left(\xi+A^{*-1} q_{j-1}\right) \overline{H_{i}\left(\xi+A^{*-1} q_{k-1}\right)}=\sum_{i=1}^{|\operatorname{det}(A)|} H_{i}\left(\xi+A^{*-1} q_{j-1}\right) \overline{H_{i}\left(\xi+A^{*-1} q_{k-1}\right)} \\
= & h\left(\xi+A^{*-1} q_{j-1}\right) \overline{h\left(\xi+A^{*-1} q_{k-1}\right)} \underbrace{\left(\sum_{i=1}^{|\operatorname{det}(A)|} e_{q_{i-1}}\left(\xi+A^{*-1} q_{j-1}\right) \overline{e_{q_{i-1}(}\left(\xi+A^{*-1} q_{k-1}\right)}\right)}_{=: s_{j-1, k-1}}
\end{aligned}
$$

Now we need to establish that $s_{j, k}=0$ for all $j \neq k$.

$$
\begin{aligned}
s_{j, k} & =\sum_{i=1}^{|\operatorname{det}(A)|} e_{q_{i-1}}\left(\xi+A^{*-1} q_{j}\right) \overline{e_{q_{i-1}}\left(\xi+A^{*-1} q_{k}\right)} \\
& =\sum_{i=1}^{|\operatorname{det}(A)|} e_{q_{i-1}}\left(A^{*-1} q_{j}-A^{*-1} q_{k}\right)=\sum_{i=0}^{|\operatorname{det}(A)|-1} e^{2 \pi i\left\langle q_{i}, A^{*-1}\left(q_{j}-q_{k}\right)\right\rangle}
\end{aligned}
$$

Since $j \neq k$, we have $q_{j}-q_{k} \neq 0$ and $A^{*-1}\left(q_{j}-q_{k}\right) \notin \mathbb{Z}^{d}$. Therefore, $e_{A^{*-1}\left(q_{j}-q_{k}\right)}$ is not the identity character on the quotient group $\mathbb{Z}^{d} /\left(A^{*} \mathbb{Z}^{d}\right)$ and by Lemma $4.6, s_{j, k}=0$. This completes the proof of the claim $\mathbf{H H}^{*}=\mathbf{I}$. 
One concrete realization of the construction described in Example 5.1 is obtained by using the so-called 'Root Raised Cosine' filter as a low pass filter. Here, we use the dyadic dilation matrix to define the dilation operator. The low pass filter is defined by

$$
H_{0}(\xi)=\left\{\begin{array}{cl}
1 & \text { for }|\xi|<3 / 20 \\
\sqrt{\frac{1+\cos \left(10 \pi|\xi|-\frac{3 \pi}{2}\right)}{2}} & \text { for } 3 / 20<|\xi|<1 / 4 \\
0 & \text { for }|\xi|>1 / 4
\end{array}\right.
$$

Hence, the Fourier transform of the refinable function $\phi$ is given by

$$
\widehat{\phi}(\xi)=\left\{\begin{array}{cl}
1 & \text { for }|\xi|<3 / 10 \\
\sqrt{\frac{1+\cos \left(5 \pi|\xi|-\frac{3 \pi}{2}\right)}{2}} & \text { for } 3 / 10<|\xi|<1 / 2 . \\
0 & \text { for }|\xi|>1 / 2
\end{array}\right.
$$

The high pass filters are given by

$$
H_{i}(\xi)=\frac{e_{q_{i-1}}}{2^{d / 2}}\left\{\begin{array}{cl}
0 & \text { for }|\xi|<3 / 20 \\
\sqrt{\frac{1-\cos \left(10 \pi|\xi|-\frac{3 \pi}{2}\right)}{2}} & \text { for } 3 / 20<|\xi|<1 / 4 . \\
1^{2} & \text { for }|\xi|>1 / 4
\end{array}\right.
$$

and, finally, the wavelets are defined in the frequency domain as follows:

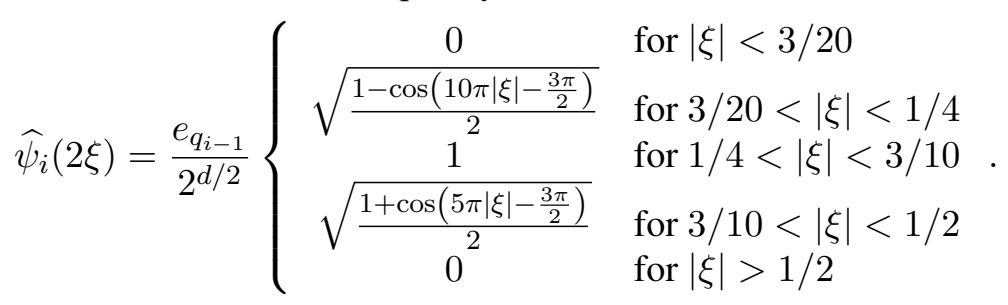

Example 5.2. In this example we produce dual frame pairs using the Mixed Extension Principle (Theorem 4.4) and Theorem 4.10. As in the previous example, all the wavelets are isotropic and the refinable functions are radial. Let $\frac{1}{2 a^{2}}<b_{2}<b_{1}<b_{0}<\frac{1}{2 a}$. Pick the analysis low-pass filter, a smooth, $\mathbb{Z}^{d}$-periodic function $H_{0}^{a}$ satisfying the following three properties:

- $H_{0}^{a}=1$ inside the ball of radius $b_{2}$

- $H_{0}^{a}=0$ on $\mathbb{T}^{d} \backslash B\left(0, b_{1}\right)$

- $\left.H_{0}^{a}\right|_{B\left(0, b_{1}\right)}$ is radial.

Similarly to the previous example, we define $\phi^{a}$ by $\widehat{\phi^{a}}=H_{0}^{a}\left(A^{*-1}\right.$.) $\chi_{\mathbb{T}^{d}}$. Now, let $h^{a}$ be a $\mathbb{Z}^{d}$-periodic function defined by,

$$
h^{a}(\xi)=\frac{1-H_{0}^{a}(\xi)}{|\operatorname{det}(A)|^{1 / 2}} .
$$

Using $h^{a}$ we define the analysis high pass filters

$$
H_{i}^{a}(\xi):=e_{q_{i-1}}(\xi) h^{a}(\xi), \quad i=1, \ldots,|\operatorname{det}(A)| .
$$

Next, let the synthesis low pass filter, a smooth, $\mathbb{Z}^{d}$-periodic function $H_{0}^{s}$ satisfying the following three properties:

- $H_{0}^{s}=1$ inside the ball of radius $b_{1}$

- $H_{0}^{s}=0$ on $\mathbb{T}^{d} \backslash B\left(0, b_{0}\right)$

- $\left.H_{0}^{s}\right|_{B\left(0, b_{0}\right)}$ is radial.

To define $|\operatorname{det}(A)|$ synthesis high pass filters $H_{i}^{s}, i=1, \ldots,|\operatorname{det}(A)|$, we use a smooth, $\mathbb{Z}^{d}$-periodic function $h^{s}$ satisfying the following three properties:

- $h^{s}=0$ inside the ball of radius $b_{3}$, for some $b_{3}>0$ 
- $h^{s}=\frac{1}{|\operatorname{det}(A)|^{1 / 2}}$ on $\mathbb{T}^{d} \backslash B\left(0, b_{2}\right)$

- $\left.h^{s}\right|_{\mathbb{T}^{d}}$ is radial

and take $H_{i}^{s}=e_{q i-1} h_{s}$. Notice, for each $i=0, \ldots,|\operatorname{det}(A)|$, the pairing of the analysis and synthesis filters. Let $\mathbf{H}_{a}$ and $\mathbf{H}_{s}$ denote the modulation matrices corresponding to the filters $H_{i}^{a}$ and $H_{i}^{s}$ respectively. (Recall that a modulation matrix is defined by Eq. (12)). We claim that $\mathbf{H}_{s} \mathbf{H}_{a}^{*}=\mathbf{I}$. To prove the claim we begin by observing

$$
\left(\mathbf{H}_{s} \mathbf{H}_{a}^{*}\right)_{1,1}(\xi)=\sum_{i=0}^{|\operatorname{det}(A)|} H_{i}^{s}(\xi) \overline{H_{i}^{a}(\xi)}=H_{0}^{a}(\xi)+|\operatorname{det}(A)| \frac{1-H_{0}^{a}(\xi)}{|\operatorname{det}(A)|}=1 \quad \text { for all } \xi \in \mathbb{T}^{d} .
$$

Now,

$$
\left(\mathbf{H}_{s} \mathbf{H}_{a}^{*}\right)_{i+1, i+1}(\xi)=\left(\mathbf{H}_{s} \mathbf{H}_{a}^{*}\right)_{1,1}\left(\xi+A^{*-1} q_{i-1}\right)=1 \text { for all } \xi \in \mathbb{T}^{d}, i=1, \ldots,|\operatorname{det}(A)| .
$$

To establish that the off-diagonal entries are zero, observe that the supports of $H_{0}^{s}\left(.+A^{*-1} q_{i-1}\right)$ and $\overline{H_{0}^{a}\left(.+A^{*-1} q_{j-1}\right)}$ which implies

$$
H_{0}^{s}\left(\xi+A^{*-1} q_{i-1}\right) \overline{H_{0}^{a}\left(\xi+A^{*-1} q_{j-1}\right)}=0 \text { for all } \xi \in \mathbb{T}^{d}, i \neq j .
$$

Hence,

$$
\begin{aligned}
\left(\mathbf{H}_{s} \mathbf{H}_{a}^{*}\right)_{j, k}(\xi) & =\sum_{i=0}^{|\operatorname{det}(A)|} H_{i}^{s}\left(\xi+A^{*-1} q_{j-1}\right) \overline{H_{i}^{a}\left(\xi+A^{*-1} q_{k-1}\right)} \\
& =\sum_{i=1}^{|\operatorname{det}(A)|} H_{i}^{s}\left(\xi+A^{*-1} q_{j-1}\right) \overline{H_{i}^{a}\left(\xi+A^{*-1} q_{k-1}\right)} \\
& =\overline{h^{a}\left(\xi+A^{*-1} q_{k-1}\right)} \underbrace{\left(\sum_{i=1}^{|\operatorname{det}(A)|} e_{\left.q_{i-1}\left(\xi+A^{*-1} q_{j-1}\right) \overline{e_{q_{i-1}}\left(\xi+A^{*-1} q_{k-1}\right)}\right)}\right.}_{=: s_{j-1, k-1}}
\end{aligned}
$$

Recall, from Example 5.1 that $s_{j, k}=0$ for all $j \neq k$. Now, the proof of the claim $\mathbf{H}_{s} \mathbf{H}_{a}^{*}=\mathbf{I}$ is complete.

Our next objective is to show that $\left\{D^{j} T_{k} \psi_{i}^{a}: j \in \mathbb{Z}, k \in \mathbb{Z}^{d}, i=1, \ldots,|\operatorname{det}(A)|\right\}$ is a Bessel sequence. For a fixed $i$ and a fixed $j$, we have,

$$
\begin{aligned}
& \sum_{k \in \mathbb{Z}^{d}}\left|\left\langle f, D^{j} T_{k} \psi_{i}^{a}\right\rangle\right|^{2}=\sum_{k \in \mathbb{Z}^{d}} \frac{1}{|\operatorname{det}(A)|^{j}}\left|\int_{\mathbb{R}^{d}} \widehat{f}(\xi) \overline{e_{k}\left(A^{*-j} \xi\right) \widehat{\psi_{i}^{a}}\left(A^{*-j} \xi\right)} d \xi\right|^{2} \\
& =\sum_{k \in \mathbb{Z}^{d}} \frac{1}{|\operatorname{det}(A)|^{j}}\left|\sum_{l \in \mathbb{Z}^{d}} \int_{A^{* j} \mathbb{T}^{d}} \widehat{f}\left(\xi+A^{* j} l\right) \overline{e_{k}\left(A^{*-j} \xi\right) \widehat{\psi_{i}^{a}}\left(A^{*-j} \xi+l\right)} d \xi\right|^{2}
\end{aligned}
$$

The sum over $l \in \mathbb{Z}^{d}$ in the last equality reduces to a sum over $l \in F$, where $F$ is a finite subset of $\mathbb{Z}^{d}$ which remains fixed for all $j$, because $\widehat{\psi_{i}^{a}}$ has compact support. Now, by applying Plancherel's theorem to

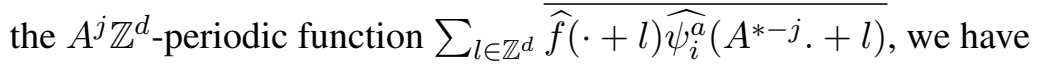

$$
\begin{gathered}
\sum_{i=1}^{|\operatorname{det}(A)|} \sum_{j=-\infty}^{\infty} \sum_{k \in \mathbb{Z}^{d}}\left|\left\langle f, D^{j} T_{k} \psi_{i}^{a}\right\rangle\right|^{2}=\sum_{i=1}^{|\operatorname{det}(A)|} \sum_{j=-\infty}^{\infty} \int_{A^{* j} \mathbb{T}^{d}}\left|\sum_{l \in F} \widehat{f}\left(\xi+A^{* j} l\right) \widehat{\widehat{\psi}_{i}^{a}}\left(A^{*-j} \xi+l\right)\right|^{2} d \xi \\
\leq \sum_{i=1}^{|\operatorname{det}(A)|} \sum_{j=-\infty}^{\infty} \int_{A^{* j} \mathbb{T}^{d}}\left[\sum_{l \in F}\left|\widehat{f}\left(\xi+A^{* j} l\right)\right|^{2}\right]\left[\sum_{l^{\prime} \in F}\left|\widehat{\psi_{i}^{a}}\left(A^{*-j} \xi+l^{\prime}\right)\right|^{2}\right] d \xi
\end{gathered}
$$




$$
\begin{array}{ll}
= & \sum_{i=1}^{|\operatorname{det}(A)|} \sum_{j=-\infty}^{\infty} \sum_{l \in F} \int_{A^{* j}\left(\mathbb{T}^{d}+l\right)}|\widehat{f}(\eta)|^{2}\left[\sum_{l^{\prime} \in F}\left|\widehat{\psi_{i}^{a}}\left(A^{*-j} \eta-l+l^{\prime}\right)\right|^{2}\right] d \eta \\
\leq & \sum_{i=1}^{|\operatorname{det}(A)|} \sum_{j=-\infty}^{\infty} \sum_{l \in F} \int_{A^{* j}\left(\mathbb{T}^{d}+l\right)}|\widehat{f}(\eta)|^{2}\left[\sum_{q \in F-F}\left|\widehat{\psi_{i}^{a}}\left(A^{*-j} \eta+q\right)\right|^{2}\right] d \eta \\
\leq & \quad \int_{\mathbb{R}^{d}}|\widehat{f}(\eta)|^{2} \sum_{i=1}^{|\operatorname{det}(A)|} \sum_{j=-\infty}^{\infty}\left[\sum_{q \in F-F}\left|\widehat{\psi_{i}^{a}}\left(A^{*-j} \eta+q\right)\right|^{2}\right] d \eta
\end{array}
$$

Using the definition of the $\psi_{i}^{a}$,s one can easily establish

$$
\sum_{j=-\infty}^{\infty}\left|\widehat{\psi_{i}^{a}}\left(A^{*-j} \eta+q\right)\right|^{2} \leq \frac{2}{|\operatorname{det}(A)|}
$$

for every $q$ which implies that $\left\{D^{j} T_{k} \psi_{i}^{a}: j \in \mathbb{Z}, k \in \mathbb{Z}^{d}, i=1, \ldots,|\operatorname{det}(A)|\right\}$ is a Bessel sequence. Similarly, we can show that $\left\{D^{j} T_{k} \psi_{i}^{s}: j \in \mathbb{Z}, k \in \mathbb{Z}^{d}, i=1, \ldots,|\operatorname{det}(A)|\right\}$ is a Bessel sequence too. Now, Theorem 4.4 implies that the families $\left\{D^{j} T_{k} \psi_{i}^{a}: j \in \mathbb{Z}, k \in \mathbb{Z}^{d}, i=1, \ldots,|\operatorname{det}(A)|\right\} \quad$ and $\left\{D^{j} T_{k} \psi_{i}^{s}: j \in \mathbb{Z}, k \in \mathbb{Z}^{d}, i=1, \ldots,|\operatorname{det}(A)|\right\}$ are a pair of dual frames for $L^{2}\left(\mathbb{R}^{d}\right)$. Also, by Theorem 4.10, the families $X_{\phi \psi}^{a}$ and $X_{\phi \psi}^{s}$ are a pair of dual frames for $L^{2}\left(\mathbb{R}^{d}\right)$.

It is easily verified that if the synthesis high pass filters in Example 5.2 are replaced by $H_{i}^{s}=e_{q i-1} \chi_{\mathbb{T}^{d}}$, the identity, $\mathbf{H}_{s} \mathbf{H}_{a}^{*}=\mathbf{I}$, still holds, but $\left\{D^{j} T_{k} \psi_{i}^{s}: j \in \mathbb{Z}, k \in \mathbb{Z}^{d}, i=1, \ldots,|\operatorname{det}(A)|\right\}$ and $X_{\phi \psi}^{s}$ are not Bessel sequences. This shows we need the hypotheses that both affine families generated from the wavelets $\psi^{a}$ and $\psi^{s}$ in Theorem 4.4 and similarly the families $X_{\phi \psi}^{a}$ and $X_{\phi \psi}^{s}$ in Theorem 4.10 are Bessel sequences.

One concrete realization of the construction described in Example 5.2 is again obtained by using the socalled 'Raised Cosine' filter as a low pass filter. Once again, we use the dyadic dilation matrix to define the dilation operator. The analysis and synthesis low pass filters are defined by

$$
\begin{gathered}
H_{0}^{a}(\xi)=\left\{\begin{array}{cl}
1 & \text { for }|\xi|<1 / 8 \\
\frac{1+\cos \left(12 \pi|\xi|-\frac{3 \pi}{2}\right)}{2} & \text { for } 1 / 8<|\xi|<5 / 24 \\
0 & \text { for }|\xi|>5 /<24
\end{array}\right. \\
H_{0}^{s}(\xi)=\left\{\begin{array}{cl}
1 & \text { for }|\xi|<5 / 24 \\
\frac{1+\cos \left(\frac{240 \pi}{11}|\xi|-\frac{50 \pi}{11}\right)}{2} & \text { for } 5 / 24<|\xi|<1 / 4 \\
0 & \text { for }|\xi|>1 / 4
\end{array}\right.
\end{gathered}
$$

Hence the Fourier transform of the scaling functions are given by the formulae,

$$
\begin{gathered}
\widehat{\phi^{a}}(\xi)=\left\{\begin{array}{cl}
1 & \text { for }|\xi|<1 / 4 \\
\frac{1+\cos \left(6 \pi|\xi|-\frac{3 \pi}{2}\right)}{2} & \text { for } 1 / 4<|\xi|<5 / 12 \\
0 & \text { for }|\xi|>5 / 12
\end{array}\right. \\
\widehat{\phi^{s}}(\xi)=\left\{\begin{array}{cl}
1 & \text { for }|\xi|<5 / 12 \\
\frac{1+\cos \left(\frac{120 \pi}{11}|\xi|-\frac{50 \pi}{11}\right)}{2} & \text { for } 5 / 12<|\xi|<1 / 2 . \\
0 & \text { for }|\xi|>1 / 2
\end{array}\right.
\end{gathered}
$$

Define the analysis and synthesis high pass filters by

$$
\begin{aligned}
& H_{i}^{a}(\xi)=\frac{e_{q_{i-1}}}{2^{d / 2}}\left\{\begin{array}{cl}
0 & \text { for }|\xi|<1 / 8 \\
\frac{1-\cos \left(12 \pi|\xi|-\frac{3 \pi}{2}\right)}{2} & \text { for } 1 / 8<|\xi|<5 / 24 \\
1 & \text { for }|\xi|>5 / 24
\end{array}\right. \\
& H_{i}^{s}(\xi)=\frac{e_{q_{i-1}}}{2^{d / 2}} \cdot\left\{\begin{array}{cl}
0 & \text { for }|\xi|<1 / 16 \\
\frac{1-\cos (16 \pi|\xi|-\pi)}{2} & \text { for } 1 / 16<|\xi|<1 / 8 \\
1 & \text { for }|\xi|>1 / 8
\end{array}\right.
\end{aligned}
$$


and finally, the wavelets are defined in the frequency domain as follows,

$$
\begin{aligned}
& \widehat{\psi_{i}^{a}}(2 \xi)=\frac{e_{q_{i-1}}}{2^{d / 2}}\left\{\begin{array}{cl}
0 & \text { for }|\xi|<1 / 8 \\
\frac{1-\cos \left(12 \pi|\xi|-\frac{3 \pi}{2}\right)}{2} & \text { for } 1 / 8<|\xi|<5 / 24 \\
1 & \text { for } 5 / 24<|\xi|<1 / 4 \\
\frac{1+\cos \left(6 \pi|\xi|-\frac{3 \pi}{2}\right)}{2} & \text { for } 1 / 4<|\xi|<5 / 12 \\
0 & \text { for }|\xi|>5 / 12
\end{array}\right. \\
& \widehat{\psi_{i}^{s}}(2 \xi)=\frac{e_{q_{i-1}}}{2^{d / 2}}\left\{\begin{array}{cl}
0 & \text { for }|\xi|<1 / 16 \\
\frac{1-\cos (16 \pi|\xi|-\pi)}{2} & \text { for } 1 / 16<|\xi|<1 / 8 \\
1 & \text { for } 1 / 8<|\xi|<5 / 12 \\
\frac{1+\cos \left(\frac{120 \pi}{11}|\xi|-\frac{50 \pi}{11}\right)}{2} & \text { for } 5 / 12<|\xi|<1 / 2 \\
0 & \text { for }|\xi|>1 / 2
\end{array} .\right.
\end{aligned}
$$

The two previous examples presented typical IMRA-wavelets and refinable functions, but there are other possible cases. Particularly, some of the wavelets constructed by means of Theorem 3.1 in [2] or in [55] can be derived from singly generated IMRAs and thus shown to be implementable with IMRA-fast wavelet algorithms. The radial wavelets of Epperson and Frazier [31,32] cannot be derived from singly generated IMRAs although they are very similar to the radial wavelets constructed in the previous two examples. The decompositions of $L^{2}\left(\mathbb{R}^{d}\right)$ for which these wavelets are used are not at all similar to those induced by $X_{\phi \psi}$ and their associated dual families.

\section{Approximation Properties of IMRAS}

In this section we explore the approximation order of the zero-resolution subspaces of IMRAs and the closed linear span of $\left\{T_{n} \phi:, n \in \mathbb{Z}^{d}\right\}$ where $\phi$ is a refinable radial function. Following the approximation theory terminology, e.g. [27], subspaces $V_{0}$ of the latter sort are called principal. Recall IMRAs core subspaces may not be singly generated, so their approximation order cannot be estimated from results applicable to principal shift-invariant subspaces only. This need motivates Proposition 6.1. If $\mathcal{S}$ is a closed subspace of $L^{2}\left(\mathbb{R}^{d}\right), \alpha>0$ and $\mathcal{S}^{\alpha}:=\{g(\cdot / \alpha): g \in \mathcal{S}\}$, then $\mathcal{S}$ has approximation order $k$ if for every $f$ in the Sobolev space $W_{2}^{k}\left(\mathbb{R}^{d}\right)$ we have

$$
\inf \left\{\|f-h\|: h \in \mathcal{S}^{\alpha}\right\} \leq C_{\mathcal{S}} \alpha^{k}\|f\|_{W_{2}^{k}\left(\mathbb{R}^{d}\right)} .
$$

Recall, that $W_{2}^{k}\left(\mathbb{R}^{d}\right)$ is the subspace of $L^{2}\left(\mathbb{R}^{d}\right)$ which contains all functions $f$ satisfying

$$
\int_{\mathbb{R}^{d}}|\hat{f}(\xi)|^{2}\left(1+\|\xi\|^{2}\right)^{k} d \xi<\infty
$$

and for $f \in W_{2}^{k}\left(\mathbb{R}^{d}\right)$ we define $\|f\|_{W_{2}^{k}\left(\mathbb{R}^{d}\right)}:=\left(\int_{\mathbb{R}^{d}}|\hat{f}(\xi)|^{2}\left(1+\|\xi\|^{2}\right)^{k} d \xi\right)^{1 / 2}$. Since, the parameter $\alpha$ in the definition of the approximation order is continuous and the scale $j$ is discrete, we use the convention $\alpha=2^{j}$, with $j \in \mathbb{Z}$ for MRAs.

We're interested in the approximation order of MRAs because it gives a crude estimate of the approximation error of a function having relatively mild smoothness based on the density of the sampling grid (completely determined by the scale $j$ ). Sharp estimates of this error are critical for image compression, however is not as critical for texture segmentation as we are only characterizing regions, not reproducing the image.

The radial refinable functions $\phi$ in Section 3 are not compactly supported. Following [27], we employ an auxiliary function

$$
\Lambda_{\phi, k}(\xi):=|\xi|^{-k} \sqrt{1-\frac{|\widehat{\phi}(\xi)|^{2}}{\sum_{l \in \mathbb{Z}^{d}}|\hat{\phi}(\xi+l)|^{2}}}, \xi \in \mathbb{T}^{d}
$$


to estimate the approximation order of a subspace $V_{0}$ which is the closed linear span of $\left\{T_{n} \phi:, n \in \mathbb{Z}^{d}\right\}$. Here we use the convention $\frac{|\widehat{\phi}(\xi)|^{2}}{\sum_{l \in \mathbb{Z}^{d}}|\hat{\phi}(\xi+l)|^{2}}=0$ if both the numerator and the denominator of this fraction vanish. According to Theorem 1.6 of [27] the approximation order of $V_{0}$ is $k$ if and only if $\Lambda_{\phi, k} \in L^{\infty}\left(\mathbb{T}^{d}\right)$. In fact, the essential boundedness of $\Lambda_{\phi, k}$ in neighborhoods of the origin determines the approximation order of $V_{0}$. Notice in Ex. 5.1 and $5.2 \hat{\phi}$ is equal to 1 in a neighborhood of the origin, forcing the restriction of $\Lambda_{\phi, k}$ to vanish on this neighborhood. This is true for every $k$, so the $V_{0}$ in Examples 5.1 and 5.2 have any approximation order. The same result holds for refinable radial functions $\phi$ whose Fourier transform has the properties described in these two examples and does not necessarily vanish outside of the fundamental domain $\mathbb{T}^{d}$.

Now we explore the approximation order of the subspaces $V_{0}$ of the non-singly generated IMRAs. Recall from Theorem 2.1, that such $V_{0}$ are of the form $P W_{\Omega}$, where $\Omega$ is a radial set. We prove the following result:

Proposition 6.1. Let $\left\{V_{j}\right\}_{j \in \mathbb{Z}}$ be an IMRA with dilations defined by the powers of a radially expansive matrix $A$ with dilation factor $a$ and $V_{0}=P W_{\Omega}$ where, $\Omega$ contains a neighborhood of the origin and is radial. If $\rho:=\sup \{r>0: B(0, r) \subseteq \Omega\}$, then

$$
\forall f \in W_{2}^{k}\left(\mathbb{R}^{d}\right), \inf _{g \in V_{j}}\|g-f\| \leq a^{-j k} \rho^{-k}\|f\|_{W_{2}^{k}\left(\mathbb{R}^{d}\right)} .
$$

Proof. It follows that $B(0, \rho) \subseteq \Omega$ and this is the biggest ball contained in $\Omega$. Let $k>0$ be an integer and pick $f \in W_{2}^{k}\left(\mathbb{R}^{d}\right)$. Then,

$$
\inf _{g \in V_{j}}\|f-g\|_{L^{2}\left(\mathbb{R}^{d}\right)}^{2}=\int_{\mathbb{R}^{d}}|\widehat{f}(\xi)-\widehat{g}(\xi)|^{2} d \xi=\int_{A^{j} \Omega}|\widehat{f}(\xi)-\widehat{g}(\xi)|^{2} d \xi+\int_{\mathbb{R}^{d} \backslash A^{j} \Omega}|\widehat{f}(\xi)|^{2} d \xi .
$$

To achieve the infimum in the LHS of the previous equations take $\widehat{g}(\xi)=\chi_{A^{j} \Omega} \widehat{f}(\xi)$. Hence,

$$
\inf _{g \in V_{j}}\|f-g\|_{L^{2}\left(\mathbb{R}^{d}\right)}^{2}=\int_{\mathbb{R}^{d} \backslash A^{j} \Omega}|\widehat{f}(\xi)|^{2} d \xi \leq \int_{\mathbb{R}^{d} \backslash a^{j} B(0, \rho)}|\widehat{f}(\xi)|^{2} d \xi=\int_{\|\xi\|>a^{j} \rho}|\widehat{f}(\xi)|^{2} d \xi .
$$

But, $\|\xi\|>a^{j} \rho$ is equivalent to $\left\|\frac{\xi}{a^{j} \rho}\right\|>1$ which implies

$$
\int_{\|\xi\|>a^{j} \rho}|\widehat{f}(\xi)|^{2} d \xi \leq \int_{\mathbb{R}^{d}}\left[\left(\frac{1}{a^{j} \rho}\right)^{2}+\left\|\frac{\xi}{a^{j} \rho}\right\|^{2}\right]^{k}|\widehat{f}(\xi)|^{2} d \xi=a^{-2 j k} \rho^{-2 k} \int_{\mathbb{R}^{d}}\left(1+\|\xi\|^{2}\right)^{k}|\widehat{f}(\xi)|^{2} d \xi .
$$

Remark 6.2. We cannot remove the assumption that $\Omega$ contains a neighborhood of the origin (a.e.): Consider $\Omega$ from Example 2.6. This particular $\Omega$ does not contain a neighborhood of the origin. In this case,

$$
\Lambda_{\chi_{\Omega}, k}(\xi):=|\xi|^{-k} \chi_{A}, \quad \xi \in \mathbb{T}^{d}
$$

is not essentially bounded for each $k>0$. Then the IMRA defined in this example does not have a positive approximation order.Also note the proof of the previous proposition does not require that $\Omega$ is radial and that $\left\{V_{j}\right\}_{j \in \mathbb{Z}}$ is an IMRA. It only requires that $\Omega$ contains a neighborhood of the origin. Therefore, we have proved a stronger result than Proposition 6.1, which is more general than Lemma 3.8 in [27].

\section{IMRA AND TEXTURE SEGMENTATION}

By segmentation of an image, we mean the construction of a partition of the image into labeled regions. Typically the number of labels is small, and the final goal is to arrive at a partition where regions having the same label are 'spatially similar' in some problem dependent sense. For example, we may wish to segment a medical image into regions of texture that describe similar tissues, where each label is associated with a type of tissue. The following discussion is kept at an intuitive level.

In the present paper we are concerned only with the multiresolution (or multiscale) signal representation as a tool enabling segmentation algorithms, for example the Digital Tissue Staining Algorithm (DTSA) 
$[15,57]$. In segmentation, the only purpose in transforming the original signal is to improve clustering, i.e. accentuate the most relevant information inherent in that signal to allow for more accurate segmentation. Details on our IMRA-approach to texture segmentation for tissue discrimination and on the application of our method to x-ray CT 3D-data sets can be found in $[15,57]^{1}$.

The IMRA approach has two significant practical advantages. First, the isotropy of IMRA filters does not introduce any directional bias, which is effective for processing the types of common medical tissues we are interested in here that do not occur with prevalent orientations (see Fig. 1). Secondly, transforms are performed in the native dimensionality of the data, furthermore the IMRA allows for efficient cascade-type implementation utilizing the discrete Fourier transform in two and three dimensions $[15,57]$.

At this point a little notation will be needed. Constraining the discussion to three dimensions (2D images are approached similarly), we consider images $f$ in $L^{2}\left(\mathbb{R}^{3}\right)$. For each pixel $z \in \mathbb{R}^{3}$ we want to associate a feature vector belonging to a fixed finite dimensional space $V$, with a norm arising from an inner product $\|\cdot\|$ on $V$. We define a bounded linear transformation $\mathcal{J}$ associating to each $f$ the $V$-valued function $\mathcal{J} f$ defined on $\mathbb{R}^{3}$. The values $\mathcal{J} f(z)$ are the feature vectors corresponding to $f$. Thus, $V$ is also referred to as the feature vector space and $\mathcal{J}$ as the feature map. The boundedness hypothesis corresponds to a robustness property of the feature map $\mathcal{J}$ : small perturbations of pixel intensities for the image $f$ generate small perturbations of the features $\mathcal{J} f$.

This very general setting allows us to begin to characterize classes of representations with desirable properties to enable successful segmentation of images, more particularly to address features of the images arising from particular applications and distinguishing features that we need to capture.

In order to segment tissues in medical images we wish to construct texture identification and associated segmentation algorithms which remain invariant under rigid motions of the image. This follows from the observation of particular tissues of interest occurring with arbitrary orientation. We let $G L(V)$ be the group of invertible linear maps of the finite-dimensional space $V$ into itself. The group $G$ of rigid motions of $\mathbb{R}^{3}$ is generated by translations and rotations and acts naturally on images $f$ via $\mathcal{R} f(z)=f\left(R^{T} z\right)$, where $R \in G$. The following definition captures the property we are interested in:

Definition 7.1. (Steerability) [3] Consider a bounded linear feature mapping $\mathcal{J}$ as above, generating for each image $f$ and each voxel $z$ in $\mathbb{R}^{3}$ a feature vector $\mathcal{J} f(z)$ in the Euclidean space $V$. We shall say that $\mathcal{J}$ is a steerable feature mapping if $\mathcal{J}$ is bounded and there is a group-homomorphism $U$ from the group $G$ of rigid motions into the general linear group $G L(V)$ of $V$ such that for each rigid motion $R$ of $\mathbb{R}^{3}$, the invertible transformation $U(R)$ verifies

$$
\mathcal{J}[\mathcal{R} f](z)=U(\mathcal{R})\left[\mathcal{J} f\left(R^{T}(z)\right)\right] \quad z \in \mathbb{R}^{3} .
$$

Take a radial refinable function $\phi$ as in Example 5.1 and let $\psi=\psi_{0}$ be the radial wavelet defined via Eqs. (27) and (28). Then, define

$$
\mathcal{J} f(z)=\left(<f, 8 T_{4 z} \phi(\cdot / 4)>, \quad<f, 2^{3 / 2} T_{2 z} \psi(\cdot / 2)>, \quad<f, T_{z} \psi>\right) \quad z \in \mathbb{R}^{3} .
$$

Alternatively one can choose analysis refinable function and associated radial wavelet as in Example 5.2. According to Definition 7.1 In [3] we prove that $\mathcal{J}$ is a bounded linear feature mapping which is not steerable. In particular, one can easily see that steerability fails for translations while if $R$ is a rotation the radiality of $\phi$ and $\psi$ imply the steerability of $\mathcal{J}$ with respect to 3D-rotations. Notice that $V$ is three dimensional in this case. We can certainly add more dimensions to $V$ but we have experimentally observed that this does not improve discrimination between various textures, primarily due to the down-sampling process. We remark that each coordinate of $\mathcal{J}$ is calculated by averaging 'locally' the image with a radial window. Intuitively, this amounts to 'low passing' local angular oscillations in an image.

It is worth noting that it is the properties of the resulting segmentation we are targeting, not the underlying representation (feature vector space) on which the segmentation algorithm will act. If the representation itself has directional variance, it is clearly much more difficult to construct effective algorithms that will not

\footnotetext{
${ }^{1}$ In $[3,57]$ the IMRA is referred to as First Generation IMRA
} 
be sensitive to direction. A particular problem arises in the form of directional selectivity of representations constructed via tensor products of lower dimensional wavelet bases. In contrast to representations derived from tensor product constructions, the feature mapping $\mathcal{J}$ defined in Eq. (31) has uniform response to every direction since $\phi$ and $\psi$ are both radial.

Segmentation algorithms based on representations that are designed originally for one-dimensional signals applied to two- or three-dimensional image segmentation (via tensor product approaches) are prone to local distortions due to variation in orientation. This is due to the fact that such tensor product constructions will tend to emphasize certain directions (they are directionally selective). Figure 3 demonstrates this claim using a standard two dimensional image known as 'Barbara' in the literature. The distortion in the various

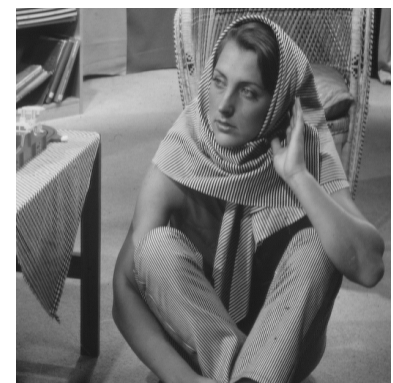

(a)

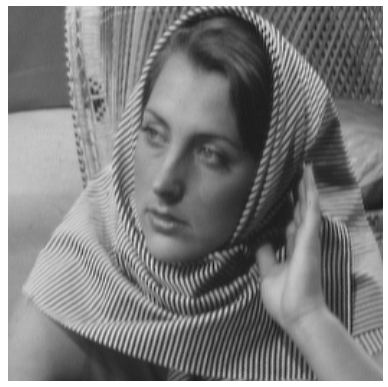

(d)

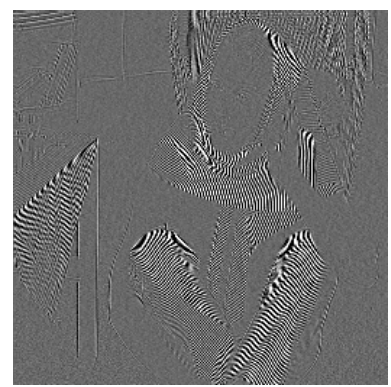

(b)

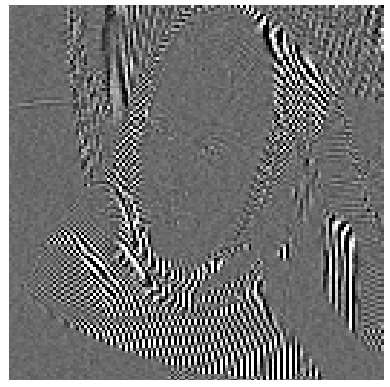

(e)

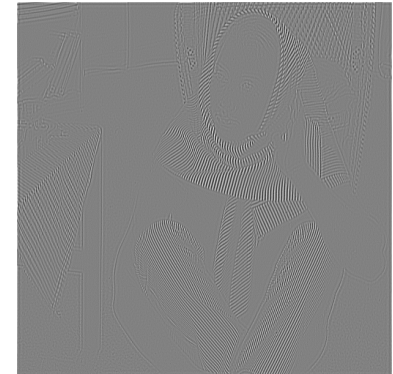

(c)

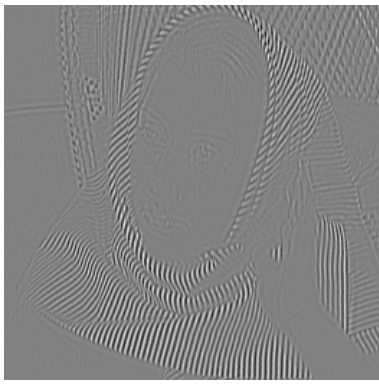

(f)

FIGURE 3. Standard 'Barbara' image a). For the same spatial region, we show the coefficient magnitude of a tensor product Daubechies- 8 wavelet basis high pass bands (i.e., the energy across all subbands rescaled to make it easier to view) in b). Similarly, the magnitude of an IMRA high-pass band is shown in c). Details of a)-c) are show in d)-f).

textures of this image can be seen in comparing 3-b to 3-c, and the associated detailed views. The Moire patterns showing up in the tensor product orthonormal wavelet representation are representative of the local distortions we mean. It is instructive to think in terms of texture patches, i.e. in a rough sense pieces of the image that are small, but large enough to identify the texture in them. Considering the woman's scarf: In the 2D-wavelet orthonormal basis, the local characteristics of such a patch vary with the orientation whereas in the IMRA coefficients, the texture is visually similar regardless of orientation (much like the original but this also has the advantages of multiscale representation). It is important to note that the orthonormal wavelet basis results shown here are typical of tensor product orthonormal wavelet basis in $2 \mathrm{D}$, since this is a fundamental effect of the tensor product. A similar effect is anticipated with non-separable 2D-wavelets without a high degree of axial symmetries. It is also important not to be mislead by the directionality of the textures shown here - after all, we are interested in 'isotropic' textures, not highly (locally) directional as in this example. We coin a definition of 'isotropic' textures in the context of tissues in [57].

Figure 3 illustrated the preferred directions in the tensor-product approach, a property of the transform, which will effect any texture. However, the effect is much easier to see in this highly-directional example. 
It is worth reiterating that Figure 3 is in no way a segmentation result, but rather serves to illustrate a fundamental difficulty that segmentation algorithms face from directionality preference of the underlying representation of the data. This is also the reason that a $2 \mathrm{D}$ example is used, even though our driving application is in 3D (also where the differences are much more subtle).

Compared to the tensor product representation, the IMRA is effective in the undistorted representation of textures regardless of local variations of orientation within the texture. We do not yet have a theoretical understanding and of why this is occurring with the IMRA-representation as Figure 3 demonstrates neither do we have a rigorous formulation of the concept 'local variations of orientation within a texture'. However, this empirically verifiable property of the IMRA-representation seems to be the strength of our Digital Tissue Staining Algorithm (DTSA) in performing isotropic soft-tissue characterization, with good empirical results. A detailed presentation of this application is beyond the scope of this paper and can be found in [15,57]. The empirical results of a clinical study utilizing CT 3D-data sets of coronary artery specimens exhibiting isotropic tissue types are reported in [38].

Fundamentally our argument is that the IMRA representation gives in this respect (i.e. directional selectivity) a better feature vector space in which texture segmentation algorithms can be applied, or at least for textures with similar properties to those discussed. Furthermore, we are working towards fully understanding the problem of steerability, and characterizing the sorts of representations that match or approximate this important property in 3D. The IMRA, the DTSA algorithm, and other ongoing work all represent steps in this direction.

\section{ACKNOWLEDGMENTS}

This research was supported in part by the following grants: Total E\&P, USA Inc., NSF-DMS 0406748, a subcontract from the University of Texas Health Science Center's 'T5'-grant and by a Research Innovation Grant by the Texas Learning and Computation Center of the University of Houston. The authors thank Professors B.G. Bodmann and I.A. Kakadiaris for many helpful discussions and Professors D.J. Kouri and D.K. Hoffman for motivating and encouraging us to study radial filters and multidimensional imaging.

\section{REFERENCES}

[1] E.H. Adelson, E. Simoncelli, and R. Hingoranp. Orthogonal pyramid transforms for image coding. In Visual Communications and Image Processing II, volume 845 of Proceedings SPIE, pages 50-58, 1987.

[2] A. Aldroubi, C. Cabrelli, and U. Molter. Wavelets on irregular grids with arbitrary dilation matrices and frame atoms on $L^{2}\left(R^{d}\right)$. Appl. Comput. Harmon. Anal, 17(2), 2004. Special issue: Frames in Harmonic Analysis, Part II.

[3] S.K. Alexander, R. Azencott, and M. Papadakis. Isotropic multiresolution analysis for 3D-textures and applications in cardiovascular imaging. In D. Van Der Ville, V. Goyal, and M. Papadakis, editors, Wavelets XII, volume 6701, pages 67011S-1 to 67011S-12. SPIE, 2007.

[4] M. Antonini, M. Barlaud, and P. Mathieu. Image coding using lattice vector quantization of wavelet coefficients. In IEEE Internat. Conf. Acoust. Signal Speech Process., pages 2273-2276, 1991.

[5] A. Ayache. Construction de bases d'ondelettes orthonormées de $L^{2}\left(R^{2}\right)$ non séparables, à support compact et de régularité arbitrairement grande. Comptes Rendus Académie des Sciences de Paris, 325:17-20, 1997.

[6] A. Ayache. Construction of non separable dyadic compactly supported wavelet bases for $L^{2}\left(R^{2}\right)$ of arbitrarily high regularity. Revista Matematica Iberoamericana 15, 15(1):37-58, 1999.

[7] A. Ayache. Some methods for constructing non separable, orthonormal, compactly supported wavelet bases. Appl. Comput. Harmon. Anal., 10:99-111, 2001.

[8] A. Ayache, E. Belogay, and Y. Wang. Orthogonal lifting: constructing new (symmetric) orthogonal scaling functions. Personal communication, 2002.

[9] L.W. Baggett, P.E.T. Jorgensen, K.D. Merrill, and J.A. Packer. Construction of Parseval wavelets from redundant filter systems. J. Math. Phys., 46:083502-1 to 083502-28, 2005.

[10] E. Belogay and Y. Wang. Arbitrarily smooth orthogonal nonseparable wavelets in $R^{2}$. SIAM Journal of Mathematical Analysis, 30:678-697, 1999.

[11] J. J. Benedetto and J. R. Romero. The construction of $d$ - dimensional multiresolution analysis frames. J. Appl. Func. Anal., 2(4):403-426, 2007.

[12] J.J. Benedetto and S. Li. The theory of multiresolution analysis frames and applications to filter banks. Appl. Comput. Harmon. Anal., 5:389-427, 1998. 
[13] J.J. Benedetto and O.M. Treiber. Wavelet frames: Multiresolution analysis and the unitary extension principle. In L. Debnath, editor, Wavelet Transforms and Time-Frequency Signal Analysis, pages 3-36. Birkhauser, Boston MA, 2001.

[14] B. Bodmann, M. Papadakis, and J. Romero. An isotropic multiresolution analysis in multidimensions. work in progress, 2008.

[15] B.G. Bodmann and M. Papadakis et. al. Frame isotropic multiresolution analysis for micro CT scans of coronary arteries. In M. Papadakis, A. Laine, and M. Unser, editors, Wavelets XI, volume 5914, pages 59141O/1-12. SPIE, 2005.

[16] M. Bownik. The structure of shift invariant subspaces of $L^{2}\left(\mathbb{R}^{n}\right)$. J. Funct. Anal., 177:282-309, 2000.

[17] Marcin Bownik. A characterization of affine dual frames in $L^{2}\left(\mathbb{R}^{n}\right)$. App. and Comp. Harm. Anal., 8:203-221, 2000.

[18] C. Cabrelli, C. Heil, and U. Molter. Self-Similarity and Multiwavelets in Higher Dimensions, volume 170 of Memoirs, Amer. Math. Soc. AMS, 2004.

[19] E.J. Candes and D.L. Donoho. Ridgelets: a key to higher dimensional intermittency? Phil. Trans. R. Soc. London, A:24952509, 1999.

[20] J. Canny. A computational approach to edge detection. IEEE Trans. Pattern Anal. Machine Intell., 36:961-1005, September 1986.

[21] O. Christensen. Frames and Riesz bases. Birkhäuser, 2005.

[22] J.M. Coggins and A.K. Jain. A spatial filtering approach to texture analysis. Pattern Recognition Letters, 3(3):195-203, 1985.

[23] A. Cohen and I. Daubechies. Nonseparable bidimensional wavelet bases. Revista Matematica Iberoamericana, 9:51-137, 1993.

[24] L. Condat, B. Forster-Heinlein, and D. Van Der Ville. A new family of rotation-covariant wavelets on the hexagonal lattice. In D. Van Der Ville, V. Goyal, and M. Papadakis, editors, Wavelets XII, volume 6701, pages 67010B-1 to 67010B-9. SPIE, 2007.

[25] J.B. Conway. A Course in Operator Theory, volume 21 of Graduate Studies in Mathematics. American Mathematical Society, Providence, RI, 2000.

[26] I. Daubechies, B. Han, A. Ron, and Z. Shen. Framelets: MRA-based constructions of wavelet frames. Appl. Comput Harmon. Anal., 14(1):1-46, 2003.

[27] C. de Boor, R. DeVore, and A. Ron. Approximation from shift-invariant subspaces of $L_{2}\left(R^{d}\right)$. Trans. Amer. Math. Soc., 341:787-806, 1994.

[28] J. Derado. Nonseprable, compactly supported interpolating refinable functions with arbitrary smoothness. Appl. Comput. Harmon. Anal., 10(2):113-138, 2001.

[29] L. Dettori and A.I. Zayed. Texture identification of tissues using directional wavelet, ridgelet and curvelet transforms. In D.R. Larson, P.R. Massopust, Z. Nashed, M.-C. Nguyen, M. Papadakis, and A.I. Zayed, editors, Frames and Operator Theory in Analysis and Signal Processing, volume 451 of Contemp. Mathem., pages 89-118. Amer. Math. Soc., 2008.

[30] D.L. Donoho and Xiaoming Huo. Beamlets and multiscale image analysis. In T.J. Barth, T. Chan, and R. Haimes, editors, Springer Lecture Notes in Computational Science and Engineering, volume 20, pages 149-196, 2002.

[31] J. Epperson and M. Frazier. An almost orthogonal radial wavelet expansion for radial distributions. J. Fourier Anal. Applic., 1(3):311-353, 1995.

[32] J. Epperson and M. Frazier. Polar wavelets and associated Littlewood-Paley theory. Dissertationes Mathematicae, 348:1-51, 1996.

[33] M. Fickus and D.G. Mixon. Isotropic moments over integer lattices. Appl. Comp. Harmon. Anal., 2008. to appear.

[34] M. Fickus, G.S. Seetharaman, and M.E. Oxley. Multiscale moment transforms over the integer lattice. In D. Van Der Ville, V. Goyal, and M. Papadakis, editors, Wavelets XII, volume 6701, pages 67011N-1 to 67011N-8. SPIE, 2007.

[35] B. Forster, T. Blu, , D. Van De Ville, and M. Unser. Shift-invariant spaces from rotation-covariant functions. Applied Comp. Harmon. Anal., 25(2):240-265, 2008.

[36] W.T. Freeman and E.H. Adelson. The design and use of steerable filters. IEEE Trans. Pattern Anal. Machine Intell., 13(9):891906, 1991.

[37] S. D. Gertz, P. Cherukuri, B. G. Bodmann, G. Gladdish, W. T. Wilner, J. L. Conyers, I. Aboshady, M. Madjid, D. Vela, S. Lukovenkov, M. Papadakis, D. J. Kouri, R. Mohammadi Mazraeshahi, L. Frazier, D. Elrod, J. T. Willerson, and S. W. Casscells. Usefulness of multi-detector computed tomography for non-envasive evaluation of coronary arteries in asymptomatic patients. Am. J. Cardiology, 97:287-293, 2006.

[38] S.D. Gertz, B.G. Bodmann, D. Vela, M. Papadakis, I. Aboshady, P. Cherukuri, S.K. Alexander, D.J. Kouri, S. Baid, A.A. Gittens, G.W. Gladish, J.L. Conyers, D.D. Cody, L. Gavish, R.M. Mazraeshahi, W.T. Wilner, L. Frazier, M. Madjid, A. Zarrabi, S. Lukovenkov, A. Ahmed, J.T. Willerson, , and S.W. Casscells. Three-dimensional isotropic wavelets for post-acquisitional extraction of latent images of atherosclerotic plaque components from micro-computed tomography of human coronary arteries. Academic Radiology, 17:1509-1519, 2007.

[39] K. Grochenig and W. Madych. Multiresolution analysis, Haar bases and self-similar tilings. IEEE Trans. Inform. Theory, 38:558-568, 1992.

[40] K. Guo, D. Labate, W. Lim, G. Weiss, and E. Wilson. Wavelets with composite dilations and their MRA properties. Appl. Comput. Harmon. Anal., 20, 2006.

[41] Bin Han. On dual tight wavelet frames. Appl. Comput. Harmon. Anal., 4(4):380-413, 1997. 
[42] W. He and M. J. Lai. Examples of bivariate nonseparable compactly supported orthonormal continuous wavelets. In M. Unser A. Aldroubi, A. Laine, editor, Wavelet Applications in Signal and Image Processing IV, volume 3169 of Proceedings SPIE, pages 303-314, 1997.

[43] P. Heinlein, J. Drexl, and W. Schneider. Integrated wavelets for enhancement of microcalcifications in digital mamography. IEEE Trans. Med. Imag., 22(3):402-413, 2003.

[44] E. Hernández and G. Weiss. A First Course on Wavelets. CRC Press, Boca Raton, FL, 1996.

[45] A.K. Jain and F. Farrokhnia. Unsupervised texture segmentation using Gabor filters. IEEE Trans. Pattern Anal. Machine Intell., 18(2):1167-1186, 1991.

[46] S. Jain, M. Papadakis, and E. Dussaud. Explicit schemes in seismic migration and isotropic multiscale representations. In E. Grinberg, D.R. Larson, P.E.T. Jorgensen, P. Massopust, G. Olafsson, E.T. Quinto, and B. Rubin, editors, Radon Transforms, Geometry, and Wavelets, volume 464 of Contemp. Mathem., pages 177-200. Amer. Math. Soc., 2008.

[47] A. Karasaridis and E. Simoncelli. A filter design technique for steerable pyramid image transforms. In Acoustics Speech and Signal Processing. ICASP, May 7-10 1996. Atlanta, GA.

[48] N. Kingsbury. Image processing with complex wavelets. Phil. Trans. R. Soc. London A, 357:2543-2560, 1999.

[49] J. Kovačević and M. Vetterli. Nonseparable multidimensional perfect reconstruction filter-banks. IEEE Trans. Inform. Theory, 38:533-555, 1992.

[50] D. Labate, W. Lim, G. Kutyniok, and G. Weiss. Sparse multidimensional representation using shearlets. In M. Papadakis, A. Laine, and M. Unser, editors, Wavelets XI, volume 5914 of SPIE Proceedings, pages 247-255, jan 2005.

[51] W. Lawton and H.L. Resnikoff. Multidimensional wavelet bases. Technical report, Aware, Inc., Bedford, MA, February 1991.

[52] P. D. Lax. Translation invariant spaces. Acta Math., 101:163-178, 1959.

[53] M. Lindemann. Approximation Properties of Non-Separable Wavelet Bases With Isotropic Scaling Matrices And Their Relation to Besov Spaces. PhD thesis, Universitaet Bremen, 2005.

[54] D. Marr. Vision, A Computational Investigation into the Human Representation and Processing of Visual Information. W.H. Freeman and Co., New York, NY, 1982.

[55] G. Olafsson and D. Speegle. Wavelets, wavelet sets and linear actions on $\mathbb{R}^{n}$. In C. Heil, P. Jorgensen, and D. Larson, editors, Wavelets, Frames and Operator Theory, volume 345 of Contemporary Mathematics, pages 253-281, 2004.

[56] M. Papadakis. Frames of translates and the generalized frame multiresolution analysis. In K. Kopotun, T. Lyche, and M. Neamtu, editors, Trends in Approximation Theory, Innovations in Applied Mathematics, pages 353-362. Vanderbilt University Press, Nashville, TN, 2001.

[57] M. Papadakis, B.G. Bodmann, S.K. Alexander, D. Vela MD, S. Baid, A.A. Gittens, D.J. Kouri, S.D. Gertz MD, S. Jain, J.R. Romero, X. Li, P. Cherukuri, D.D. Cody, G. W. Gladish MD, I. Aboshady MD, J.L. Conyers, and S.W. Casscells. Texturebased tissue characterization for high-resolution CT-scans of coronary arteries. Comm. in Numer. Methods in Engineering, 2009. to appear.

[58] M. Papadakis, G. Gogoshin, I.A. Kakadiaris, D.J. Kouri, and D.K. Hoffman. Non-separable radial frame multiresolution analysis in multidimensions. Numer. Function. Anal. Optimization, 24:907-928, 2003.

[59] J. Portilla, V. Strela, M.J. Wainwright, and E.P. Simoncelli. Image denoising using Gaussian scale mixtures in the wavelet domain. IEEE Trans. Image Processing, 12(11):1338-1351, 2003. Computer Science techn. rep. nr. TR2002-831, Courant Institute of Mathematical Sciences.

[60] N. Rajpoot, R. Wilson, and Z. Yao. Planelets: A new analysis tool for planar feature extraction. In Proceedings 5th International Workshop on Image Analysis for Multimedia Interactive Services (WIAMIS'04), Lisbon, Portugal, April 2004.

[61] A. Ron and Z. Shen. Affine system in $L_{2}\left(R^{d}\right)$ : The analysis of the Analysis operator. Journal Func. Anal., pages 408-447, 1997.

[62] I.W. Selesnick. Smooth wavelet tight frames with zero moments. Appl. Comput. Harmon. Anal., 10(2):163-181, 2001.

[63] I.W. Selesnick and L. Sendur. Iterated oversampled filter banks and wavelet frames. In M. Unser A. Aldroubi, A. Laine, editor, Wavelet Applications in Signal and Image Processing VIII, volume 4119 of Proceedings of SPIE, 2000.

[64] E.P. Simoncelli, W.T. Freeman, E.H. Adelson, and D.J. Heeger. Shiftable multi-scale transforms. IEEE Trans. Inform. Theory, 38(2):587-607, 1992.

[65] J.-L. Starck, Y. Moudden, J. Bobin, M. Elad, and D.L. Donoho. Morphological component analysis. In M. Papadakis, A.F. Laine, and M. Unser, editors, Wavelets XI, volume 5914, pages 59140Q-1 to 59140Q-15. SPIE, 2005.

[66] G. Tzagkarakis, B. Beferull-Lozano, and P. Tsakalides. Rotation-invariant texture retrieval with Gaussianized steerable pyramids. IEEE Trans. Image Processing, 15(9):2702-2718, 2006.

[67] M. Vetterli and J. Kovačević. Wavelets and Subband Coding. Prentice Hall PTR, Englewood Cliffs, NJ, 1995.

[68] Y. Wan and R.D. Nowak. Quasi-circular rotation invariance in image denoising. In ICIP, volume 1, pages 605-609, 1999.

[69] R.L. Wheeden and A. Zygmund. Measure and Integral. An Introduction to Real Analysis. Pure and Applied Mathematics. Marcel-Dekker Inc., 1977. 